EMPIRICAL IMPLICATIONS OF ALTERNATIVE MODELS

OF FIRM DYNAMICS

\author{
Ariel Pakes \\ Richard Ericson
}

Working Paper No. 2893

\author{
NATIONAL BUREAU OF ECONOMIC RESEARCH \\ 1050 Massachusetts Avenue \\ Cambridge, MA 02138 \\ March 1989
}

Discussions with Buz Brock, Gary Chamberlain, Art Goldberger, and Boyan Jovanovic have been very helpful. Steve Berry provided first rate programming assistance. This research was funded by the NSF (grant SES8520261) and the Graduate School of the University of Wisconsin. All errors remain, of course, our own responsibility. This paper is part of NBER's research program in Productivity. Any opinions expressed are those of the authors not those of the National Bureau of Economic Research. 
NBER Working Paper \#2893

March 1989

\section{EMPIRICAL IMPLICATIONS OF ALTERNATIVE MODELS OF FIRM DYNAMICS}

\section{ABSTRACT}

This paper considers two models for analyzing the dynamics of firm behavior that allow for heterogeneity among firms, idiosyncratic (or firmspecific) sources of uncertainty, and discrete outcomes (exit and/or entry). Models with these characteristics are needed for the structural econometric analysis of several economic phenomena, including the behavior of capital markets when there are significant failure probabilities, and the analysis of productivity movements in industries with large amounts of entry and exit. In addition, these models provide a means of correcting for the self-section induced by liquidation decisions in empirical studies of firms responses to alternative policy and environmental changes. It is shown that the two models have different nonparametric implications - implications that depend only on basic behavioral assumptions and mild regularity conditions on the functional forms of interest (one distinction between them corresponds to the distinction between heterogeneity and an ergodic form of state-dependence; a form in which the effect of being in a state in a particular period erodes away as time from that period lapses). The nonparametric implications enable the construction of testing and selection correction procedures that are easy to implement (they do not require the computationally difficult, and functional-form specific, estimation algorithms that have been used to empirically analyze stochastic control models with discrete outcomes in the past). The paper concludes by checking for the implications of the two models on an eight-year panel of Wisconsin firms. We find one model to be consistent with the data for retail trade.

\section{Ariel Pakes}

Department of Economics

P.O. Box 1972, Yale Station

Yale University

New Haven, CT 06520
Richard Ericson

Department of Economics

Columbia University

New York, NY 10027 


\section{Introduction}

This paper considers the empirical implications of two models of the dynamics of $f$ irm behavior that allow for heterogeneity anong firms, idiosyncratic (or firm-specific) sources of uncertainty, and discrete events (exit and/or entry). Our reason for providing an expirical framework with these features are twofold. Pirst, the nature of uncertainty, and its relationship to exit and/or entry, is at the heart of several issues ve, as economists, try to analyze. Examples include the analysis of capital markets when there are diverse possible outcome paths and significant failure probabilities; the evolution of the size distribution of the firms in an industry; and the analysis of industry supply (or productivity) changes when ore efficient firms thrive and grow, and less efficient contract and, in the extreme case, exit. The second reason for studying models that allow for uncertainty and exit is that some allowance has to be made for these phenomena before ve can get an accurate empirical picture of firms' responses to any policy or enviornmental change. Table 1 illustrates why this is so.

The table provides information on the fraction of firms operating in visconsin in 1978 that vere liquidated by 1986 (more details on the data will be given in Section 5). Firas are classified as liquidated only if they physically closed down (changes of ownership are treated separately). If we vere to use these data to build a panel of firms to follow the impact of some (say) policy change, ve would, at lesst traditionally, start from the 1978 cross-section and then construct the panel by elininating those firms not in operation over the entire eight-year period. Coluan 5 shows that this procedure would lose a third of the firms due to liquidations, and column 6 shows that this third would account for about a fifth of the 
jobs in 1978. If we decided to consider only the larger of the 1978 firms, say those with more than 50 employees (and as column 7 shows, this is a selection which, by itself, omits over a third of the 1978 jobs), liquidation would be somewhat less prevalent, but would still cause an attrition rate of about 15 percent. The last two rows of the table give an indication of the extent of changes in ownership in this data (this includes mergers and acquisitions). To the extent that the pre and post change firms cannot be spliced together, changes in ownership also generate attrition. It is a relatively more important source of attrition among larger firms, but even if we confine ourselves to firms with over 50 employees, and assume that all the changes in ownership result in attrition, changes of ownership vould still only account for 40 percent of total attrition (liquidation accounts for the rest). Note that, when taken together, liquidations and changes of ownership vould cause the attrition of almost half the firms in the 1978 sample, and of about a quarter of those with more than 50 employees.

If liquidation decisions were independent of the economic phenomena typically being investigated, then the omission of the liquidated firms from the sample might lead to an imprecise, but would not lead to an inconsistent, description of the phenomena of interest. This is, however, hardly likely. Firms terminate their activities when they perceive adverse changes in the distribution of their future profit streams. The phenomena we typically want to investigate involve the actual profitability (and productivity) changes resulting from alternative policy and environmental changes. If there is any relationship at all between perceptions and realizations we will, by eliminating those firms which liquidate, omit precisely those firms for whom the events in question are likely to have 
had a particularly negative impact. That is, we will tend to omit one tail of the distribution of responses we set out to study. 1

To control for the selection induced by the liquidation process ve need a model that explains why firms operating in sinilar environents develop differently - a model with idiosyncratic outcones that allow for exit. It least two such models are currently available, and each will, no doubt, prove more useful in approxinating the characteristics of different industries in different time periods. This paper provides a simple set of procedures which enable the researcher to deternine whether either of them might be relevant for the problem at hand.

The first model considered here is a model with passive Bayesian learning. Firms are endowed at birth with an unknown value of a time-invariant profitability parameter which determines the distribution of its profits thereafter. Past profit realizations contain information on the value of the parameter which determines the distribution of possible future profit streams, and this fact is used by management to form a probability distribution over future net cash flows (see Jovanovic, 1982). The second model is a model of active exploration. It assumes that the firm knows the current value of the parameter that determines the distribution of its profits, but that the value of that profitability parameter changes over time in response to the stochastic outcones of the firm's own investments, and those of other actors in the same market (see Ericson and Pakes, 1989). In both models firms act so as to maximize the expected discounted value of future net cash flow, and in both cases optimal behavior generates a set of stopping states; i.e. outcomes which, if realized, would induce the firm to exit. Moreover, both models are 'complete' in the sense that if we vere villing to append a set of precise 
functional form assumptions to them, they would produce framevorks rich enough to take directly to data.

The strategy of appending precise functional forn assumptions and then using their implications to structure the data, is the strategy taken in all of the recent econometric literature on analyzing stochastic control models involving discrete outcomes (see Iiller, 1984; Volpin, 1984; Pakes, 1986; and Rust, 1987). Its success depends upon, anong other diverse factors, the extent of prior information on the relevance of alternative assumptions. Ve eschew it here because there is not a great deal of a information on either which of the nodels (if any) is appropriate for different data sets or on the relevance of alternative functional form assumptions. Yoreover, just as in all the previous literature on discrete choice optimal stochastic control models, were we to estimate fully parametric versions of these models we vould have to build a different -. ion algorithm for each form estinated. This makes it difficult, if impossible, to examine the robustness of the najor empirical results to crlanges in the specification of the model.

The alternative strategy we choose is to look for empirical implications of the different models that depend only on the models' basic behavioral assumptions, and some mild regularity conditions on the relevant functional forms. Precisely because these 'nonparanetric' implications have to be valid for a variety of functional form, they cannot require onal form specific estimation and testing algorithms. Consequently, inere are computationally simple ways of checking whether they are consistent with the data. Therefore, in addition to not being dependent on particular functional form assumptions, our strategy is easy to implement. On the other hand, the nonparametric procedures provided here do not 
produce precise values for alternative response parameters. Their goals are only to provide a low cost, easily interpretable, characterization of the data which suffices to: 1) distinguish which, if either, of the alternative models seens relevant for the problen at hand, and 2) act as a basis for building a procedure for correcting for the selection problen induced by the liquidation process when one of the nodels seens appropriate.

One of the nonparametric differences between the two models corresponds to the distinction between heterogeneity and state dependence that has played so large a role in labor econonetrics (see lecknan, 1981; Chamberlain, 1984; and Beckman and Singer; 1984). In particular the passive learning model implies that the stochastic process generating the size of a firm is characterized by a generalized form of heterogeneity, while the model with active exploration implies that this stochastic process is generated by a quite general form of state dependence. Theory restricts the state dependence in the active learning model to have ergodic characteristics; i.e. the effect of being in a state in a particular period erodes away as time from that period lapses. So we develop a test for the distinction between heterogeneity and ergodic forms of state dependence based on -mixing conditions. The test is sinple, intuitive, and seems to be able to distinguish between the two models on panel data sets the size of the ones used here (these follow about 400 observations over eight years).

In particular, we find both the -aixing test, and an analysis of the evolution of the size distribution of firms in a cohort, suggest that one model is consistent with the data for manufacturing, while the other seems consistent with the data for retail trade. The importance of this result 
is twofold. First the different models have distinctly different implications for the manner and the extent to which firm-specific uncertainties get resolved over tine, and hence for the vay in which issues related to these uncertainties ought to be analyzed. Second, the tro models imply different determinants for the probability of liquidetion, and hence different procedures for correcting for liquidation induced attrition in the analysis of firm's responses to alternative policy and environental changes.

Section 2 of the paper outlines the passive learning model and then derives its nonparametric implications. Section 3 does the same for the model with active exploration. In Section 4 ve develop appropriate estimation and testing procedures. Section 5 begins with a description of the Visconsin panel, and then examines various subsets of it for the implications of the two models. Section 6 considers further implications of the empirical results.

\section{Notation}

The distribution of any random variable, say $x$, conditional on any event, say $z$, is denoted $P_{x}(\cdot \mid z)$, and its density (with respect to the implied dominating measure) by $p_{x}(\cdot \mid z)$. Superscripts denote the vector of all prior realizations of a process, and subscripts denote a particular value, so $x^{t}=\left(x_{1}, \ldots, x_{t}\right)$. Veak vector inequalities are interpreted element by element, but a strong vector inequality means only that at least one of the element by element inequalities is strong. $z$ vill be used for the generic set, and $z$ for a member of that set. Lemmas, theorems, examples etc. will be numbered in one consecutive ordering within each section. They are referred to in the following sections with a section 
prescript.

Section 2. Passive Learning.

This section considers nodels in which each firs is endowed with a time-invariant characteristic which deternines the distribution of its profits, but whose value is not known to anagenent at the time the fin begins operation. Models of industries conposed of firms which learn about an unknown profitability parameter have been provided by Jovanovic (1982) and Lippman and Bumelt (1982). Following Jovanovic (1982), we consider a Bayesian learning process. At entry the firn believes the value of its characteristic, say $\theta$, is a random draw from sone known distribution. Each period the firm is in operation it obtains a realization from the distribution of profits conditional on the true value of its $\theta$. These realizations are used to compute a sequence of posterior distributions. The posterior available in each period is used as a basis for decision-making in that period. The decisions of interest are whether to produce at all and, if so, at what scale. If the firm does decide not to produce it sells off its assets and exits, never to reappear again. Note that in this model learning is passive in the sense that information is obtained as a costless byproduct of operating. One possible analogy is to the operation of a retail outlet. The outlet learns whether its neighborhood will support its product, and, if so, at which scale of operation.

Jovanovic (1982) focuses on establishing the existence of a perfect foresight equilibrium for a homogeneous product industry conposed of firms which operate in this manner. Ve focus on the implications of the learning process on the evolution of cohorts of firms, where cohorts are defined by 
entry dates. In particular we shall look for empirical implications that rely on the nature of the learning process, and only some mild regularity conditions on the form of the profit function and the underlying distributions of interest. Later we conpare these inplications to data in an attempt to identify those sectors in which this for of learning process seems relevant.

\subsection{The Yodel}

It will be assumed that each entrant is endowed with a value of $\theta$ which, in turn, determines the distribution of a payoff relevant random variable $\eta$, say $P_{\eta}(\cdot \mid \theta)$. To motivate our assumptions, consider the example of a homogeneous product industry of price-takers whose production efficiencies are subject to random perturbations so that profits in period $t$ are $\pi_{t}=a_{t} \eta_{t} F\left(\ell_{t}\right)-v_{t}{ }^{\prime} \ell_{t}$ where; $\ell_{t}$ is a vector of input quantities, $u_{t}$ provides their prices, $F(\cdot)$ is a concave production function, $\left\{\eta_{t}\right\}$ is a sequence of independent and identically distributed (i.i.d.) random variables, and $a_{t}$ is the product price. Issume $\eta_{t}$ is known at the time $\ell_{t}$ is chosen. Then

$$
x_{t}=x\left(\eta_{t} ; \omega_{t}, p_{t}\right)=\max _{\ell_{t}}\left\{a_{t} \eta_{t} F\left(\ell_{t}\right)-\omega_{t} \ell_{t}\right\}
$$

and $\pi\left(\eta ; \omega_{t}, p_{t}\right)$ is an increasing function of $\eta$. In a perfect foresight equilibrium future prices will be known, so that if $\theta$ were also known the distribution of future profits could be calculated directly fron $P_{\eta}(\cdot \mid \theta)$. Since management does not know $\theta$ it is assumed to sunmarize its beliefs about that parameter in terms of a probability distribution over the 
possible values of $\theta$. At entry, nanagenent only knows that $\theta$ is a random draw from $G_{0}(\theta)$. The first period produces an $\eta$ which managenent uses, together with Bayes law, to update its prior $\left[G_{0}(\theta)\right]$ and form a posterior which is then used to make second period decisions. If the fire stays in operation, this updating process continues and decisions are ade on the basis of the sequence of updated posteriors.

Is the example illustrates, the nodel will require at least four primitives; a sequence of random variables, a class of distributions for those random variables indexed by $\theta$, a prior distribution for $\theta$, and a payof function. Before introducing these primitives ve need a way of comparing distribution functions; i.e. ve need an interpretation for the statement that one value of $\theta$ is 'better than' another. Ve shall assume that the family of distributions formed from different values of $\theta$ can be ordered in the likelihood ratio sense defined below. This ensures that higher realizations of the payoff relevant $\eta$ lead to Bayesian posteriors for $\theta$ that assign larger probability to higher values of $\theta$ (see below, and Vilgrom, 1981).

1. Definition (likelihood ratio ordering, or $\lambda_{\ell_{r}}$ )

Let $P_{1}(\cdot)$ and $P_{2}(\cdot)$ be two distributions possessing densities $P_{1}(\cdot)$ and $P_{2}(\cdot)$ (with respect to some dominating aeasure), and with support, $Z^{k}$, a compact subset of $\mathbb{R}^{k}$, $k$-dimensional Euclidean space. Ve will say that $P_{1}$ likelihood ratio dominates $P_{2}$, in the strong sense, and write $P_{1} z_{l r} P_{2}$, if and only if,

$$
p_{1}\left(z_{1}\right) p_{2}\left(z_{2}\right)-p_{2}\left(z_{1}\right) p_{1}\left(z_{2}\right)>0
$$


whenever $z_{1}>z_{2}$, and $\mathrm{p}_{1}\left(z_{1}\right)$ or $\mathrm{p}_{2}\left(z_{2}\right)>0, z_{1}, z_{2} \in z^{\mathbf{k}}$. If weal inequalities replace the strong inequalities in this definition, we vill say that $P_{1}$ likelihood ratio doninates $P_{2}$ in the veak sense, and write $P_{1}>\operatorname{trk} P_{2}$.

If $P_{1} 2_{\ell r} P_{2}$ then, for any two possible values of $z$, the ratio of the probabilities of a larger to the smaller 2 value is alvays higher for $P_{1}$; i.e., $P_{1}$ is more likely to have generated the higher 2 value. 2 The following lemma points out that $z_{\ell r}$ is a stronger criteria for ordering distribution functions than the fare faliar first order stochastic - ie criteria.

2. Lenina (likelihood ratios and stochastic doninance).

Say $P_{1}$ stochastically dominates $P_{2}$, and write $P_{1}{ }_{s} P_{2}$, if and only if for every nondecreasing nonconstant function, $h(\cdot)$, such that $\int h(\zeta) P_{1}(d \zeta)<\infty$

$$
h(\zeta) P_{1}(d \zeta)>/ h(\zeta) P_{2}(d \zeta)
$$

Then,

$$
P_{1}>_{\ell r} P_{2} \text {, implies, } P_{1}>_{s} P_{2}
$$

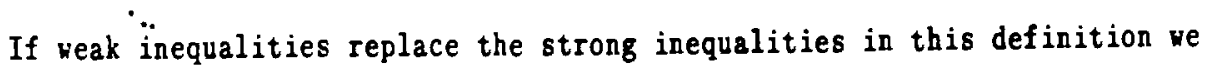
say that $P_{1}$ stochastically dominates $P_{2}$ in the weak sense, and write $P_{1}>_{s w} P_{2} \cdot P_{1}^{2} \ell T * P_{2}$ implies $P_{1} 2_{s w} P_{2}$. 
Proof See Ross (1983), Lppendix 1, 3.1, and 4.1.

Issumption 3 provides the prinitives of the passive learning nodel and endows them with some regularity conditions. It generalizes the assumptions used in our example. In particular the example assuned that conditional on a $\theta \in \theta$, the sequence of payoff relevant randon rariables, $\left\{\eta_{t}\right\}$, are independently and identically distributed (i.i.d.) over tine. Then the joint distribution of the sequence $\left\{\eta_{t}\right\}$ conditional on a $\theta \in \theta$ is entirely described by the single distribution, $P_{\eta}(\cdot \mid \theta)$. Though the i.i.d. case is easy to deal with, it produces a host of very strong enpirical implications which are a result of the i.i.d. assunption and not of the logic of the passive learning model per se. Ve, therefore, allow for dependence in the stochastic process generating $\left\{\eta_{t}\right\}$ conditional on $\theta$. In $(3 . i)$ we assume only that the marginal distribution of $\eta_{t}$ conditional on $\theta$ is stationary (does not depend on time), and that the conditional distribution of $\eta_{t}$ (conditional on past $\eta$-realizations) satisfies the condition that higher past values of $\eta$ are at least as likely to lead to higher future values of $\eta$. (3.ii) insures that higher values of $\theta$ are better in the $\ell r$-sense; $i . e$. it insures that for any $t$, higher values of the vector $\eta^{t}=\left(\eta_{1}, \ldots, \eta_{t}\right)$ are nore likely to be generated by larger $\theta$ values. (3.iv) provides the profit and size functions. It is important that both be increasing in $\eta .^{3}$

3. Issumption (primitives of the model)

(i) $\left\{\eta_{t}\right\}$ is a sequence of payoff relevant random variables (a stochastic process) whose joint distribution, say $\underline{P}(\theta)$, is indexed by a $\theta \in \theta$, where $\theta$ is a compact subset of $\mathbb{R}_{+}$. The arginal distribution of $\eta_{t}$ is 
stationary and is denoted by $\mathrm{P}_{\eta}(\cdot \mid \theta)$, while its conditional distribution satisfies a weak $\ell r-0$ rdering in realizations of $n^{t-1}$, say $n^{t-1}$ : i.e.

$$
P_{\eta_{t}}\left(\cdot \mid n_{1}^{t-1}, \theta\right)>_{\ell \Gamma W} P_{\eta_{t}}\left(\cdot \mid n_{2}^{t-1}, \theta\right)
$$

whenever $n_{1}^{t-1} \geq n_{2}^{t-1}$.

(ii) The family of distributions

$$
\mathbb{P}=\{\underline{P}(\theta): \theta \in \theta\}
$$

have marginal distributions with support $\mathbf{N}$ (a compact subset of $\mathbf{R}_{+}$) and densities with respect to some dominating measure. Further, these distributions satisfy an $\ell r$-ordering in $\theta$; i.e., provided $\theta>\theta$ ' we have, for every $t$

$$
{ }_{\eta}^{\mathrm{t}}(\cdot \mid \theta)>_{\ell I}{ }_{\eta^{\mathrm{t}}}^{\mathrm{t}}\left(\cdot \mid \theta^{\prime}\right)
$$

(iii) $G_{0}(\cdot)$ is a prior probability distribution with density $g_{0}(\cdot)$ on $\theta$.

(iv) $\pi(\cdot)$ and $S(\cdot)$ are continuous increasing functions from $N$ into $R_{+}$. $\pi(\cdot)$ provides the payoff to, and $S(\cdot)$ the size of, the firm. [ ]

Our behavioral assumption is that management acts so as to maximize the expected discounted value of future net cash flow conditional on current information, where the conditional distribution of future net cash 
flows are formed, in a Bayesian fashion, from; the family of $\eta$ processes $(\mathbb{P})$, the prior for $\theta\left[G_{0}(\cdot)\right]$, and past realizations of $\eta$, say $n^{t}=\left(n_{1}, \ldots, n_{t}\right)$. The next assumption provides these conditional distributions.

\section{Assumption [posterior distributions]}

Let $J_{t}$ contain all information available in period $t$. Then

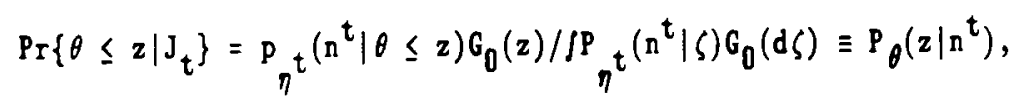

for $z \in B$. Yoreover $P_{\theta}\left(\cdot \mid n^{t}\right)$ has a density, $P_{\theta}\left(\cdot \mid n^{t}\right)$, with respect to the $G_{0}$ measure $\left(\right.$ for $n^{t} \in \mathbb{N}^{t}$, and all $\left.t\right)$.

Lemma 5 states that, under the $\ell r$-ordering assumptions, higher past $\eta$ realizations lead to more favorable posteriors for $\theta$. It follows directly from Bayes law and assumption (3.ii).4

5. Lemma (monotonicity of posteriors)

For any $t$, let $n_{1}^{t}, n_{2}^{t} \in N^{t}$ with $n_{1}^{t}>n_{2}^{t}$, then

$$
P_{\theta}\left(\cdot \mid n_{1}^{t}\right)>l r P_{\theta}\left(\cdot \mid n_{2}^{t}\right)
$$

Now consider the decision problem facing the owners of a firm which has been in existence $t$ periods and has had $\eta$ realizations of $n^{t}$. The owners must choose whether to continue in operation over the coming period, or close down and sell the firm at the value, If the ouners decide to 
operate the firm they will obtain the profits over the coning period, plus the option of keeping the firm in operation over subsequent periods should they desire to do so.5

Issume, temporarily, the existence of a bounded function, say $V_{t+1}\left(n^{t+1}\right)$, from $N^{t+1}$ into $R$, which provides the value of continuing in operation from period $t+1$ given a realization of $\eta^{t+1}$ equal to $n^{t+1}$. Then, letting $\beta \epsilon(0,1)$ be the discount factor, ve have

$$
V_{t}\left(n^{t}\right)=E\left[x\left(\eta_{t+1}\right) \mid n^{t}\right]+\beta E\left[\max \left\{\phi, V_{t+1}\left(\eta^{t+1}\right)\right\} \mid n^{t}\right],
$$

where for any $h(\cdot)$, the expectation $E\left[h\left(\eta^{t+1}\right) \mid n^{t}\right] \equiv \int h\left(\zeta, n^{t}\right) P_{\eta_{t}}\left(d \zeta \mid n^{t}\right)$.

Given (6) the optimal strategy of the owner is straightforward. Operate the firm if and only if $V_{t}\left(n^{t}\right) \geq$. Theorem 7 insures that the value function in (6) exists and then provides some of its properties.

7. Theorem (existence and montonicity of the value function)

At each $t$ there exists a unique $V_{t}(\cdot): N^{t} \rightarrow R_{+}$which provides the value of continuing in operation assuming optimal behavior in each future period. $V_{t}(\cdot)$ is bounded, satisfies (6), and is nondecreasing in $n^{t}$; i.e., if $n_{1}^{t}$ ? $n_{2}^{t}$, then $v_{t}\left(n_{1}^{t}\right) \geq v_{t}\left(n_{2}^{t}\right)$ [for $n^{t} \mathbb{N}^{t}$, and all $t$ ]

Proof See Appendix I.

Note that Theorem 7 depends only on 1 ssumption 3. It does not depend on: the precise functional form (or even the curvature) of the profit 
function (so the production function could display regions of increasing returns); on the form of $G_{0}(\cdot)$; or on the family P provided that it satisfy the monotone likelihood ratio properties in (3) (in particular the posteriors for $\theta$ need not possess sinple sufficient statistics, nor need they be veakly continuous in their argunents). Ve now move on to consider the empirical implications of the passive learning model and ve shall focus on inplications which require only the essumptions revieved above.

\subsection{Empirical Implications of Passive Learning.}

Throughout we shall focus on the enpirical iaplications of the passive learning model that are true at each age (that nodel also has liait properties as age grows large, but it is hard to use these as a basis for empirical analysis without further, a priori, inforation). Ve begin by deriving the implications of the passive learning model on the evolution of the size distribution of firms.

The theorem that underlies our results on the evolution of the size distribution is the economist's (far more palatable) version of the Darkinian dictum of "survival of the fittest." It states that as age increases the $\theta$-distribution of the surviving firms improves (in the stochastic dominance sense). This is a result of self-selection. Is time passes firms with lower $\theta$ 's are more likely to draw lower $\eta$ 's and self-liquidate.

8. Theorem (the evolution of the $\theta$-distribution) 
Let $\Lambda^{t}=\left\{n^{t}=\left(n_{1}, \ldots, n_{t}\right): V_{1}\left(n_{1}\right)>, \ldots, v_{t}\left(n^{t}\right)>\right\}$, and

$$
x_{t}\left(n^{t}\right)=\left\{\begin{array}{ll}
1 & \text { if } n^{t} \in \Lambda^{t} \\
0 & \text { if } n^{t} \in u^{t}
\end{array} .\right.
$$

Then a firm is still operating in period $t$ if and only if $x_{t}=1$. Further, for every $z \in \theta$ and all $t$ let

$$
P_{\theta}(z \mid t) \equiv \operatorname{Pr}\left\{\theta \leq z \mid \chi_{t}=1\right)
$$

Then

$$
P_{\theta}(\cdot \mid t+1) 2_{s w} P_{\theta}(\cdot \mid t)
$$

inof Take an arbitrary $(z, t)$. Then, by Bayes law,

$$
\begin{aligned}
\operatorname{P}_{\theta}(z \mid t) & =\operatorname{Pr}\left(\chi_{t}=1 \mid \theta \leq z\right) G_{0}(z) / \operatorname{Pr}\left\{\chi_{t}=1\right\} \\
= & {[\theta \leq z} \\
& \left.\operatorname{Pr}\left\{\chi_{t}=1 \mid \theta\right\} G_{0}(d \theta)\right] /\left[{ }_{\theta} \int \operatorname{Pr}\left\{\chi_{t}=1 \mid \theta\right\} G_{0}(d \theta)\right] .
\end{aligned}
$$

ve must show that $P_{\theta}(z \mid t-1) \geq P_{\theta}(z \mid t)$. For this is suffices that

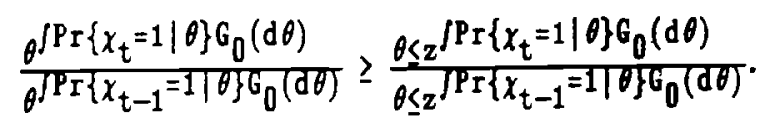

Using the fact that

$$
\operatorname{Pr}\left\{x_{t}=1 \mid \theta\right\} \equiv \operatorname{Pr}\left\{x_{t}=1 \mid x_{t-1}=1, \theta\right\} \operatorname{Pr}\left\{x_{t-1}=1 \mid \theta\right\}
$$


and letting

$$
q_{1}(d \theta)=\operatorname{Pr}\left\{x_{t-1}=1 \mid \theta\right\} G_{0}(d \theta) / \theta_{0} \operatorname{Pr}\left\{x_{t-1}=1 \mid \theta\right\} G_{0}(d \theta) \text {, and }
$$

$$
Q_{2}(d \theta)=\left[\begin{array}{ll}
0 & \text { for } \theta>z \\
\operatorname{Pr}\left\{x_{t-1}=1 \mid \theta\right\} G_{0}(d \theta) / \alpha_{z} \int \operatorname{Pr}\left\{x_{t-1}=1 \mid \theta G_{0}(d \theta),\right. & \text { otherwise }
\end{array}\right]
$$

(8.1) can be rewritten as

$$
\theta_{\theta} \operatorname{Pr}\left\{x_{t}=1 \mid x_{t-1}=1, \theta\right\} Q_{1}(d \theta) \geq{ }_{\theta} \int \operatorname{Pr}\left\{x_{t}=1 \mid x_{t-1}=1, \theta\right\} Q_{2}(d \theta) .
$$

Since (8.2) implies $Q_{1}(\cdot)>_{s w} Q_{2}(\cdot),(8.3)$ vill be true provided $\operatorname{Pr}\left\{x_{t}=1 \mid x_{t-1}=1, \theta\right\}$ is nondecreasing in $\theta$. To see that this is indeed the case wite

$$
\operatorname{Pr}\left\{x_{t}=1 \mid x_{t-1}=1, \theta\right\}=\int \operatorname{Pr}\left\{x_{t}=1 \mid n^{t-1}, \theta\right\}{ }_{\eta}{ }^{t-1}\left\{d^{t-1} \mid n^{t-1} \in \Lambda^{t-1}, \theta\right\} .
$$

Then, $\operatorname{taking} \theta \geq \theta^{\prime}$

$$
\begin{aligned}
& \int \operatorname{Pr}\left\{\chi_{\mathrm{t}}=1 \mid \mathrm{n}^{\mathrm{t}-1}, \theta\right\} \mathrm{P}_{\eta}^{\mathrm{t}-1}\left\{\mathrm{dn}^{\mathrm{t}-1} \mid \mathrm{n}^{\mathrm{t}-1} \in \Lambda^{\mathrm{t}-1}, \theta\right\} \geq \\
& \int \operatorname{Pr}\left\{\chi_{t}=1 \mid n^{t-1}, \theta^{\prime}\right\} P{ }_{\eta}^{t-1}\left\{d n^{t-1} \mid n^{t-1} \epsilon^{t-1}, \theta\right\} \geq \\
& \int \operatorname{Pr}\left\{x_{t}=1 \mid n^{t-1}, \theta^{\prime}\right\} P{ }_{\eta}^{t-1}\left\{\mathrm{dn}^{t-1} \mid \mathrm{n}^{\mathrm{t}-1} \in \Lambda^{\mathrm{t}-1}, \theta^{\prime}\right\} \text {, }
\end{aligned}
$$

where the first inequality follows from the nonotonicity of $V(\cdot)$ and the fact that $P_{\eta}\left(\cdot \mid n^{t-1}, \theta\right)$ is stochastically increasing in $\theta$, and the second 
from (3.1) and the fact that if $\mathrm{P}_{\eta}(\cdot \mid \theta){ }_{\ell}{ }_{\ell \mathrm{r}}{ }^{\mathrm{P}}{ }_{\eta}^{\mathrm{t}}\left(\cdot \mid \theta^{\prime}\right)$, then, for any $\Delta \in \mathrm{N}^{\mathrm{t}}$, $P_{\eta} \mathrm{t}\left(\cdot \mid \eta^{\mathrm{t}} \epsilon \Lambda, \theta\right)>\ell r{ }_{\eta} \mathrm{t}\left(\cdot \mid \eta^{\mathrm{t}} \epsilon \Lambda, \theta^{\prime}\right)$ (see Ross, 1982, appendix I).

Our first empirical implication of the passive learning aodel is a direct corollary of theorem 8. Since size is an increasing function of $\eta$, and $\eta$ is stochastically increasing in $\theta$, the fact that the distribution of the surviving firms is stochastically increasing over tine inplies that the size distribution of surviving firms ought to be stochastically increasing in time.

9. Corollary (The evolution of the size distribution.)

Let $x_{t}$ be defined as in Theorem 8 , recall that $S_{t}=S\left(\eta_{t}\right)$, and for all $z$ and $t$ define

$$
P_{S}(z \mid t)=\operatorname{Pr}\left\{S_{t} \leq z \mid x_{t}=1\right\}
$$

Then, provided $t \geq t$,

$$
P_{S}(\cdot \mid t)>{ }_{S H} P_{S}\left(\cdot \mid t^{\prime}\right)
$$

There are many vays of employing Corollary $\theta$ to identify industries that might abide by the passive learning model. The simplest is to plot the size distribution for different ages and compare them; the proportion of the sample greater than any given size should increase in age. Iore 
generally the corollary implies that if $h(\cdot)$ is any increasing function, then whenever $t \leq t$ '

$$
\hbar(t)=\int h(\zeta) P_{s}(d \zeta \mid t) \leq / h(\zeta) P_{8}\left(d \zeta \mid t^{\prime}\right)=h\left(t^{\prime}\right) .
$$

So ve could take the sample analogue of $h(t)$ [the sample mean of $h(S)$ ], and investigate whether it increases in age. Ye cone back to these points below. Note also that Theore (8) and Corollary ( 9 ) inply that each sequence of distribution functions, $\left\{P_{\theta}(\cdot \mid t)\right\}$, and $\left\{P_{8}(\cdot \mid t)\right\}$, converges (pointwise), to a vell-defined liniting distribution, say $P_{\theta}(\cdot \mid \omega)$ and $P_{S}(\cdot \mid x)$.

Implications of the passive learning nodel that specify a monotonic relationship between two or more observables are particularly useful since they can be checked against data without imposing undue functional form restrictions. Though the literature on the passive learning model seems to have missed Corollary 9 , it has associated at least three other monotonic relationships with passive learning. These are that:

i) the hazard rate is nonincreasing in current size; i.e., that $\operatorname{Pr}\left\{\chi_{\mathrm{t}}=0 \mid \chi_{\mathrm{t}-1}=1, \mathrm{~s}_{\mathrm{t}-1}=\mathrm{s}_{\mathrm{t}-1}\right\}$ is nonincreasing in $\mathrm{s}_{\mathrm{t}-1}$;

ii) the hazard rate is nondecreasing in age (usually, but not alvays, conditional on size);

iii) and that the variance in growth rates (again usually conditional on size) is nonincreasing in age

(these implications are discussed in Jovanovic, 1982; Evans, 1987a and 1987b; and Dunne, Roberts and Samuelson, 1987).

The next example shows that of these three only the first survives our search for nonparametric implications of the passive learaing nodel (the 
example assumes, as did Jovanovic, 1982, that the distribution of $\left\{\eta_{t}\right\}$ conditional on $\theta$ is i.i.d.). It is true, hovever, that the first implication, that is that hazard rates are nonincreasing in size at a given age, both persists and is consistent with the data from every empirical study we are aware of [Churchill, 1955; Vedervang, 1965; Bvans 1987a and 1987b; Dunne, Roberts, and Samuelson, 1987]. Iowever, wost other models that allow for mortality, including Ericson and Pakes's (1989) nodel of active exploration, also imply mortality rates that decrease in size for a given age. Therefore, this property fails to distinguish anong the alternative models, and we do not pay further attention to it in this paper.

As to the other implications, the fact that the passive learning nodel does not imply that either hazard rates, or the variance in growth rates, decline in age (at least not without further ad hoc assumptions) is somewhat disconcerting. Decreasing hazards and decreasing variances in growth rates have both been associated with the passive learning model in the past, and, in addition, have been shown to be fairly robust features of the data. On the other hand, the intuition underlying our counterexample is clear enough. For many functional forms it will take time to accumulate the information necessary to ensure that exit is optimal, and this fact generates an initial increasing portion to the hazard function (actually the example generalizes this intuition and generates a hazard function which oscillates over age). Is to differences in the variance in growth rates over age, these will depend upon, anong other factors, the relative variances of $\eta$ conditional on $\theta$ for different values of $\theta$. If $\theta$-values which are more likely to induce exit are associated with low variances, the 
observed variance in growth rates may vell increase over age.

\section{Example}

Let $\pi_{t}=\pi \cdot \eta_{t}$, with $\left\{\eta_{t}\right\}$ i.i.d. conditional on $\theta$,

$$
\eta_{t}=\left\{\begin{array}{l}
1 \text { with probability } \theta \\
0 \text { otherwise }
\end{array} ; \text { and } \theta=\left\{\begin{array}{l}
\delta \text { with probability } \ell \\
0 \text { otherwise }
\end{array} .\right.\right.
$$

The posterior for $\theta$ in this problem depends only on the couple $\left(x_{t}, t\right)$, where $x_{t}=\max \left[n_{1}, \ldots, n_{t}\right]$. Consequently the value function in (6) has the simple form,

$$
v_{t}\left(n^{t}\right)=V\left(x_{t}, t\right)
$$

$x_{t}$ is either 0 or 1 . If $x_{t}=1$ management knows that $\theta=\delta$ and a direct calculation shows

$$
\mathrm{V}(1, \mathrm{t})=\pi \delta /(1-\beta)>\mathbf{1},
$$

where the inequality is by assumption. This inequality ensures that if $x_{t}=1$ management will never drop out. If $x_{t}=0$ the firm continues in operation if and only if $V(0, t) \geq$. It is easy to show that $\operatorname{Pr}\left\{x_{t+1}=1 \mid x_{t}=0, t\right\}=\operatorname{Pr}\left\{\eta_{t+1}=1 \mid x_{t}=0, t\right\}$ decreases in $t$, and converges to zero. This ensures that $V(0, t)$ decreases in $t$ and converges to zero. Clearly then, there exists a unique $t^{*}$ such that $Y(0, t) \geq$ if and only if $\mathrm{t} \leq \mathrm{t}^{*}$ : Let $S\left(\eta_{\mathrm{t}}=1\right)=\mathrm{S}, \mathrm{S}\left(\eta_{\mathrm{t}}=0\right)=0$, $\mathbb{H}\left(\mathrm{t}, \mathrm{S}_{\mathrm{t}}\right)$ be the hazard rate for the firms of size $S_{t}$ in period $t$, and $H(t)$ be the unconditional hazard. 
Straightforward calculations show that for

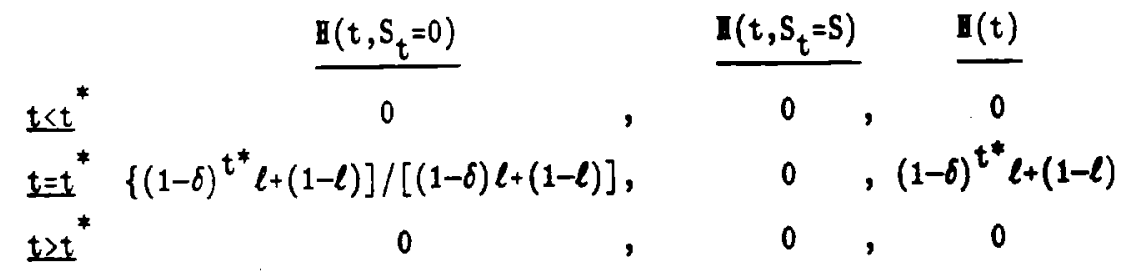

So neither the conditional, nor the unconditional, hazard declines in age.

T. ismply reflects the fact that for many possible assumptions on the relevant functional forms it will take time to gather the inforation required to decide whether exit is optinal.

Next ve consider the variance in growth rates. Provided $t>t^{*}$, any firm that is active has $\theta=\delta$, and $V\left(S_{t+1}-S_{t} \mid S_{t}\right)=V\left(S_{t+1} \mid \theta=\delta\right)=S^{2} \delta(1-\delta)$, -narad ess of $S_{t}$. If $t<t^{*}$ and $S_{t}=S$, then $\theta$ still is $\delta$ with probability and $V\left(S_{t+1}-S_{t} \mid S_{t}\right)$ is still given by the above formulae. So the a. a iance in growth rates conditioned on $S_{t}=S$ is constant over age. However, if $t<t^{*}$, and $S_{t}=0$, then $\theta$ can equal either $\delta$ or 0 with positive probability, and the variance in the growth rate is $\left[\delta S^{2}(1-\ell)(1-\delta) \ell\right] /[(1-\ell)+(1-\delta) \ell]^{2}$. Thus

$$
v\left(S_{t+1}-S_{t} \mid S_{t}=0, t>t^{*}\right) / v\left(S_{t+1}-S_{t} \mid S_{t}=0, t<t^{*}\right)=\frac{[(1-l)+(1-\delta) l]^{2}}{(1-l) l \cdot \delta}
$$

which cän be made as large as ve like by choosing $\delta$ or $l$ small enough. The variance in growth rates need not decline in age. Whether or not they do will depend upon whether grovth rates associated with high $\theta$ 's are wore 
variant than growth rates associated with low $\theta$ 's, an issue which the basic passive learning model is silent on.

To see how this example generalizes, consider the case where $\theta$ has a beta prior distribution with paraneters $(r, s)$, i.e., $G_{0}(\cdot)=B(r, s)$, so that $\theta$ can take any value between zero and one. The posterior in this case is another beta with paraneters $r+\stackrel{t}{\Sigma} \eta_{i}$ and $s+t-\Sigma_{\eta_{i}}$, so that the son, $x_{t}=\stackrel{t}{\Sigma} \eta_{i}$, and $t$, can be used as sufficient statistics. (Note that $x_{t}$ is a nonnegative integer.) Using an argument analogous to that given above we find that for any fixed $x, V(x, t)$ declines to zero with $t$. Thus for each $x$ there exists a $t^{*}(x)$ such that $V(x, t) \geqslant$ according as $t \leqslant t^{*}(x)$ [see Figure 1]. Both the mortality, and the hazard rate will be zero for a value of $t$ such that $t^{*}(x)<t<t^{*}(x+1)($ for $x=1,2, \ldots)$. Yoreover it can be shown that $t^{*}(x+1)$ cannot equal $t^{*}(x)+1$ for consecutive values of $x$. That is, the hazard function will usually have a zero between any two positive portions, making it oscillate over age. For $t=t^{*}(x)$ the hazard and mortality rates will be determined by the precise form of the prior. One such sequence of hazard rates is given in the bottom part of Figure 1. Similar pictures could be drawn for the variance in growth rates. [ ]

This example illustrates that if we are interested in other nonparametric implications of the passive learning nodel we should look beyond the implications of passive learning on the pattern of either the hazard or the variance in growth rates. It is, therefore, fortunate that the passive learning model has some very distinctive inplications on the underlying structure of the conditional probabilities generating growth and mortality. 
These implications stem primarily from the fact that $\theta$ is time-invariant. Is a result, early realizations of $\eta$ contain infornation about the parameter that deteraines the distribution of its future values; and this will be true no matter the time that elapses in the interin. Put differently, the dependence in the joint distribution of $\eta_{t}$ and $\eta_{1}$ does not erode avay as $t$ grows large. This is seen nost clearly in the special case where, conditional on $\theta$, the $\left\{\eta_{t}\right\}$ are an i.i.d. process. In this case, for any $n$ ' and $z$

$$
\begin{aligned}
& { }_{\eta^{t}}{ }\left(z \mid \eta_{k}=n^{\prime}\right)={ }_{\theta} / P_{\eta}(z \mid \theta) P_{\theta}\left(d \theta \mid \eta_{k}=n^{\prime}\right) \\
& ={ }_{\theta} / P_{\eta}(z \mid \theta) P_{\eta}\left(n^{\prime} \mid \theta\right) g_{0}(\theta) d \theta / \theta / P_{\eta}(n \mid \theta) B_{0}(\theta) d \theta ;
\end{aligned}
$$

which is independent of $t$ and $k$. This strong invariance property is destroyed when we allow $\theta$ to index the nore general family of stochastic processes permitted in (3). In the general case we have, for any $z \in \mathbb{N}$,

$$
P_{\eta_{t}}\left(z \mid \eta_{k}=n^{\prime}\right)=\int P_{\eta_{t}}\left(z \mid \eta_{k}=n^{\prime}, \theta\right) P_{\theta}\left(d \theta \mid \eta_{k}=n^{\prime}\right),
$$

and since $P_{\eta_{t}}\left(z \mid \eta_{k}=n^{\prime}, \theta\right)$ can depend upon $t$ and $k$, so can $P_{\eta_{t}}\left(z \mid \eta_{k}=n^{\prime}\right)$. However, the passive learning model does inply that the dependence in this latter distribution has two sources, one of which will not erode avay as $t$ grows large. That is, though the dependence in the process generating $\eta_{t}$ conditional on $\theta$ (in the integrand) may erode away with $t$ (it will if the process generating $\eta_{t}$ is ergodic), the dependence that results from the effect of the realization of $\eta_{\mathbf{k}}$ on the posterior for $\theta$ will not.

This argument can be formalized and then used to produce a test for 
the passive learning model based on differences between the arginal distribution of $S_{t}=S\left(\eta_{t}\right)$, and the distribution of $S_{t}$ conditional on $S_{1}$. Actually we can do better than this and produce tests based on a conparison of the distribution of $S_{t}$ conditional on $S_{t-1}, \ldots, S_{t-t}$ to the distribution of $S_{t}$ conditional on $S_{t-1}, \ldots, S_{t-k}$, and $S_{1}$, for any $k \geq 0$. Vith positive $k$ this test is likely to be more powerful against alternatives in which the value of the parameter deternining the firn's distribution of profits evolves in a larkovian fashion over tine (and one such alternative is the model of active exploration considered in the next section).

Our test is a direct inplication of the following theorea. The theorem states that if we choose any group of years for which there is information on past realizations of $\eta$, and derive the family of posterior distributions for $\theta$ conditional on possible $\eta$-realizations in those years, then members of the family with higher past $\eta$-realizations will stochastically dominate those with lover $\eta$-realizations.

11. Theorem (conditional distributions for $\eta_{t}$ )

Let $t$ and $k$ be positive integers with $t \geq k$, and $\left(i_{1}, \ldots, i_{k}\right)$ be any selection of $k$ distinct elements from $\{1, \ldots, t-1\}$. Then if $n_{1}^{\prime}=$ $\left(n_{i_{1}}^{1}, \ldots, n_{i_{k}}^{1}\right)$ and $n_{2}^{\prime}=\left(n_{i_{1}}^{2}, \ldots, n_{i_{k}}^{2}\right)$ are arbitrary $\left(i_{1}, \ldots, i_{k}\right)$ histories of $\eta$ satisfying $n_{-1}>n_{2}$, and $x_{t}$ is defined as in (8),

$$
P_{\eta_{t}}\left(\left.\cdot\right|_{-1}, x_{t}=1\right)>P_{\eta_{t}}\left(\cdot \mid \underline{n}_{-2}, x_{t}=1\right) \text {. }
$$

Proof. See Appendix I. 
The empirical implication of theoren (11) that we will be using is that it implies that for any $k \geq 0$, and any $\left(n_{t-1}, \ldots, n_{t-k}\right) \in \mathbb{N}^{k-1}$,

$$
P_{\eta_{t}}\left(\cdot \mid n_{t-1}, \ldots, n_{t-k}, n_{1}, x_{t}=1\right)>P_{\eta_{t}}\left(\cdot \mid n_{t-1}, \ldots, n_{t-k}, n_{1}^{\prime}, x_{t}=1\right),
$$

whenever $n_{1}>n_{1}^{\prime}$. Corollary (13) is an imediate implication of (12).

13. Corollary

Let $t$ and $k$ be nonnegative integers with $t>k$, and let $\chi_{t}$ be defined as in Theorem 8. Then

$$
E\left[S_{t} \mid S_{t-1}=s_{t-1}, \ldots, S_{t-k}=s_{t-k}, S_{1}=s_{1}, x_{t}=1\right]
$$

is strictly increasing in $s_{1}$ for almost every $\left(s_{t-1}, \ldots, s_{t-k}\right)$. [ ]

That is, expected future size conditional on $\mathbf{k}$ past sizes and survival will be strictly increasing in the initial size. This is because the parameter which determines the conditional distribution of the payoff relevant $\eta$ is time-invariant. In models in which these conditional distributions depend on a parameter which evolves over time in response to, say, the outcomes of a firm's exploratory investment, corollary (13) will not necessarily be true. Ve turn to these types of nodels now.

\section{Section 3. Active Exploration}

This section considers the empirical implications of a model (originally developed by Ericson and Pakes, 1989), in which firms can 
invest to improve the value of a parameter, say $\boldsymbol{v}$, which deternines the distribution of its profits. In the model with active exploration (in contrast to in the passive learning nodel) managenent is assuned to know its current value of $\omega$ (and hence the actual profit distribution it faces), and makes current production decision based on it. On the other hand $y$ itself evolves over time in response to the outcones of the firn's own investment process, and the investments of other firms operating in related markets. These outcomes are stochastic; in the active exploration model the firm invests to explore and develop alternative market niches which may, or may not, prove profitable.

In this model the distribution of futures states is deternined entirely by the current state and the optinal investment policy. It is, therefore, independent of the age of the firm per se. Startup is treated as the appearance of an idea which, given current market conditions, - arars worth exploring. Formally it is an initial location on the waxis. idea requires substantial successful development before it can -. case noticeable profits, the initial $w$ is associated with a distribution of profits which is degenerate (or nearly so) at zero. Successful investment will enable the idea to be embodied in a more profitable marketable good or service. Onsuccessful exploration nay well convince the entrepeneur that the whole idea is not vorth pursuing and lead to liquidation.

Ericson and Pakes begin with a simple nodel in which the distribution of the firm's profits depends only on the difference between the firn's own level of development and an exogenous aggregate index of the state of the industry. They then generalize to cases in which the firm's profit distribution also depends explicitly on the levels of development of all 
the encumbents in, and the potential entrants to, the industry. Here we suffice with a brief description of the simpler model, as this special case of the sore general framevork is sufficient to contrast its empirical implications to those of the passive learning nodel just described. Igain we consider only those empirical inplications that are nonparanetric in the sense that they require only mild regularity conditions on the relerant functional forms.

\section{The Active Exploration Yodel}

Ve will assume that the state space is countable and index it by the integers so that $w \in l$. Each firm operating in period $t$ is endoved with an $w_{t}$. Higher values of $w$ are better in the sense that the distribution of the payoff relevant $\eta$ is stochastically increasing in $w$. Management has three choices to make in each period, and they are ade to maximize the expected discounted value of future net cash flows. First the firm must decide whether to operate at all. If it decides against it receives a liquidation value of and exits never to reappear again. If the firm does operate management must decide on both a level of current input demand, and an amount of exploratory investment, say $x_{t}$. Given a realization of $\eta$, current input choices vill determine current operating profits, say $\pi\left(\eta_{t}\right)$. Current cash flows are

$$
R\left(\eta_{t}, w_{t}, x_{t}\right)=\pi\left(\eta_{t}\right)-c\left(w_{t}\right) x_{t}
$$

where $c(\cdot)>0$, and can be decreasing in $\omega$ to reflect the possibility that more profitable firms may find it easier to raise finance capital. Increases in current investment decrease current cash flow but make higher 
values of $w_{t+1}$, and hence higher future profits, wore likely. In particular, let $\tau_{t+1}=w_{t+1}-w_{t}$, and $J_{t}$ be the information available to management at $t$. Then we assume that for $z \in \mathbb{I}$,

$$
P_{\tau}\left(\tau_{t+1} \leq z \mid J_{t}\right)=P_{\tau}\left(z_{t} \mid x_{t}\right),
$$

where $P_{T}\left(\cdot \mid x_{t}\right)$ is stochastically increasing in $x$. Tence, to foralize the firm's decision problem ve will require the following prinitives.

1. Assumption (primitives of the active exploration model)

i) $\mathbb{P}=\left\{P_{\eta}(\cdot \mid \omega): \omega \in \mathbb{Z}\right\}$, is a family of distribution functions indexed by $\omega$. The family has support, $N$, a compact subset of containing zero, and exhibits a veak first order stochastic dominance ordering in w, i.e.

$$
P_{\eta}(\cdot \mid \omega)>_{s w} P_{\eta}\left(\cdot \mid \omega^{\prime}\right)
$$

whenever $v>v^{\prime}$. It is assumed that $\lim _{v \rightarrow \infty} P_{\eta}(0 \mid v)=1$. (This, together with the assumption that $x(0)=0$, insures that for seall enough payoffs are zero with probability arbitrarily close to one.)

ii) $\mathbb{P}_{\tau}=\left\{P_{\tau}(\cdot \mid x): x \in \mathbb{R}_{+}\right\}$is a fanily of distributions with support $T$, a compact subset of $\square$, exhibiting a weak first order stochastic doninance ordering in $x$, i.e. 


$$
P_{\tau}(\cdot \mid x)>_{S W} P_{\tau}\left(\cdot \mid x^{\prime}\right)
$$

whenever $x>x^{\prime}$, and satisfying the condition that

$$
P_{T}(0 \mid 0)=1
$$

so that the firm's product cannot be inproved without one investment. The family of densities $\left\{P_{\uparrow}(\cdot \mid x): x \in \mathbb{R}_{+}\right\}$, is (pointwise) differentiable in $x$ with derivatives which are decreasing in $x$ for $\tau>0$, and increasing in $x$ for $\tau$ $<O$ (this insures that the investment problem is concave and therefore has a unique solution), and both $p_{\tau}(0 \mid x)$ and $p_{\tau}(-1 \mid x)$ are strictly positive for all $x$ less than any finite upper bound (these are technical conditions whose roles are explained in more detail below).

iii) $x(\cdot)$ and $S(\cdot)$ are increasing functions of $\eta$, and $c(\cdot)$ is non-increasing function of $\omega$, into $\mathbb{R}_{+} \cdot \pi(\cdot)$ provides the profits, and $S(\cdot)$ provides the size, of the firm; while $c(\cdot)$ provides the cost of a unit of $x . \quad x(0)=0$, and $c(\cdot)$ is bounded away from zero.

Ve now consider management's choice of policies. Letting $v_{0}$ be the initial state and $\chi_{\tau}$ be the indicator function which takes the value one if the firm is active in period $\tau$ and zero elsewhere, a policy, say $d$, is a sequence of functions apping available information into operating and investment decisions, that is 


$$
d=\left\{x_{0}\left(J_{0}\right), x_{0}\left(J_{0}\right), x_{1}\left(J_{1}\right), x_{1}\left(J_{1}\right), \ldots\right\}
$$

with $x_{T}=x_{T}\left(\mathrm{~J}_{T}\right)$ and $x_{T}=0$ implying $x_{t+\tau}=0$ for teI,$x_{T}=x_{T}\left(J_{T}\right)$, and $=\left\{\omega_{\tau}, x_{\tau-1}, x_{\tau-1}, w_{\tau-1}, \ldots, w_{0}\right\}$. Lecall that $\mathbf{R}\left(\eta_{\tau}, w_{\tau}, x_{\tau}, x_{\tau}\right)=r\left(\eta_{\tau}\right)-c\left(\omega_{\tau}\right) x_{\text {: }}$ if $x_{\tau}=1$ and zero otherwise, so the expected discounted value of net cash flows given the policy $d$ is

$$
\left.v_{d}\left(\omega_{0}\right)=E_{d}\left\{\Sigma \beta^{T} R\left(\eta_{T}, \omega_{T}, x_{T}, x_{T}\right)+\left(x_{T-1}-x_{T}\right)\right] \mid \omega_{0}\right\}
$$

where $\beta \epsilon(0,1)$ is a discount factor, and the expectation is taken assumin that the $d$-policy is followed. Note the (1) implies that $R(\cdot)$ is bounde and let

$$
V(w)=\sup _{d} V_{d}(w)
$$

for each $\omega$. $\Delta$ policy $d^{*}$ will be optimal if $V_{d}^{*}(\omega)=V(\omega)$ for all $\omega$. If optimal policy exists management chooses it, in which case the expected discounted value of future net cash flow is $V(\omega)$. Ianagement will opera the firm if and only if $V(\omega)>$, the liquidation value. The following theorem combines the results from Ericson and Pakes (1989) that are used our derivation of the empirical implications of their model. The theore is followed by diagrammatic and verbal expositions of its contents.

2. Theorem (properties of the active exploration model).

$A$ unique optimal policy and associated value function exist and the have the following characteristics: 
i) $V(u)$ is bounded and nondecreasing in $w$.

ii) The optimal policy, $\mathbf{x}_{\tau}^{*}\left(\mathrm{~J}_{\tau}\right)$ is bounded, depends only on current $w$, and is stationary, i.e. for all $\tau$

$$
x_{\tau}^{*}\left(J_{\tau}\right)=x_{\tau}^{*}\left(w_{\tau}\right)=x^{*}\left(w_{\tau}\right) \leq \bar{x}<\omega .
$$

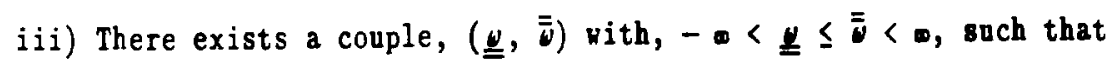

$$
x^{*}(u)=0 \text { if } u \notin\left\{w^{\prime}: \underline{\underline{w}} \leq \boldsymbol{u}^{\prime} \leq \overline{\bar{u}}\right\} \text {. }
$$

iv) There exists a second couple $(\underline{w}, \bar{w})$, with $-\infty<\underline{\omega} \leq \underline{\underline{w}} \leq \overline{\bar{u}} \leq \bar{\omega}<\infty$, such that

$$
Y(\omega)>\text { if and only if } \omega>\varphi \text {, }
$$

and

$$
\inf \left[\begin{array}{l}
\left.\inf \operatorname{Pr}\left\{\omega_{t} \leq \bar{\omega} \mid \omega_{0}\right\}\right]=1 \\
t \omega_{0} \leq \bar{\omega}
\end{array}\right.
$$

Proof: See Appendix 2.

Parts (i) and (ii) of this theoren ensure that both the value function and investment policy are stationary functions of $v$, the value function being increasing in $\omega$. Figure 2 illustrates this with one special case. In the figure $\Lambda(\omega)=\int \pi(\eta) P_{\eta}(d \eta \mid \omega)$, provides expected profits conditional on 4 . The value of $w$ below which a firm exits, i.e. the $w$ in (2.iv), is 
determined by the point at which $V(\omega)$ equals . In this example $\underline{\omega}=\underline{\underline{\omega}}$, that value of $w$ below which a firw stops investment. So positive investment occurs at $\underline{\omega}+1$, even though profits at that point are zero $\mathrm{w}$ probability one. The incentive for the investnent is that it akes highe values of $w_{t+1}$, and hence higher future profits, are likely. The noneta value of an increase in $\omega$ is $V\left(\omega_{t+1}\right)-V\left(\omega_{t}\right)$. Since $V(v)$ is bounded, aft sone point increases in 4 cannot bring with it much of a change in $V(\cdot)$. It follows that, after some $\omega$, it will not be in the firm's interest to invest at all. The $\psi$ at which this occurs is the $\overline{\bar{u}}$ of (2.iii). If $\omega>\overline{\bar{u}}$ no investment takes place and this insures (see 1.ii) that the firn's iot increase (in fact it will stochastically deteriorate as other firms gradually develop goods and services that obsolete the product of this $\mathrm{firm}$ ). Let $\tau^{*}$ be the largest value of $\tau$ that has positive probabili when $x=\bar{x}$ (recall that $\bar{x}=\max x^{*}(w)$, and that $\tau^{*}$ is finite by virtue of 1.ii). Then firms with $u_{t}<\overline{\bar{u}}$ have $v_{t+1} \leq \overline{\bar{u}}+\tau^{*}=\bar{u}$, and firas with $\overline{\bar{w}}<\omega_{t} \leq \bar{w}$ have $w_{t+1} \leq w_{t}$. So if $\omega_{t} \leq \bar{w}$, so must be $w_{t+1}$. This explains the second statement in $2 . i v$; that is, if $\omega_{0} \leq \bar{w}$, then, with probability one, so will be the entire sequence $\left\{w_{t}\right\}_{t=0}^{\infty}$.

$V(\omega)$

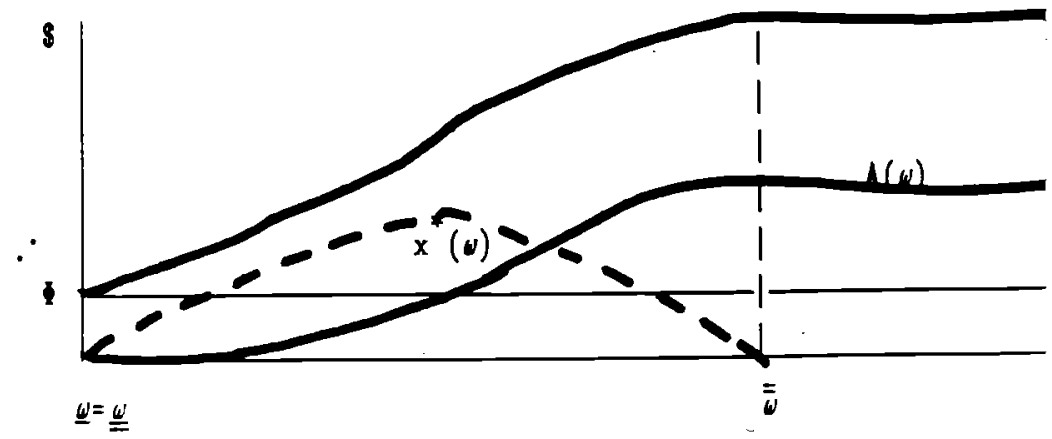

Figure 2: Policies in the Active Exploration Yodel 
Since all values of $\omega \leq \underline{\omega}$ induce permanent exit, there is no need to distinguish among them. It is, therefore, convenient to transform the state space by the map $f(\cdot)$, where

$$
f(\omega)= \begin{cases}0 & \text { for } \omega \leq \underline{\omega} \\ \omega-\underline{\omega} & \text { elsevhere. }\end{cases}
$$

Let $\mathbf{K}=\bar{w}-\underline{w}$, so that if $f\left(w_{t}\right) \leq \mathbf{x}$, so is $f\left(w_{t+1}\right)$. Ve shall work only with values of $f(\omega)$ in what follows. It the risk of some notational confusion, then, ve also label its values by $\omega$.

With this understanding, theorem 2.2 , implies that the sequence $\left\{w_{t}\right\}$ together with any $\omega_{0} \leq X$ is a finite state Yarkov chain on $n=\{0,1, \ldots, \mathbf{K}\}$. Its 'zero' or 'death' state is absorbing, so the transition matrix for the chain is given by $\underline{p}$, where

$$
\underline{p}=\left[p_{i, j}\right]=\left[\begin{array}{l}
1,0 \ldots, 0 \\
p_{i, j}
\end{array}\right]
$$

and for $0<i \leq k$

$$
p_{i, j}=\left\{\begin{aligned}
p_{\tau}\left(\tau=j-i \mid x^{*}(i)\right), & \text { for } \mathbf{x} \geq j>0 \\
\sum_{\tau \leq-i} p_{\tau}\left(\tau=j-i \mid x^{*}(i)\right), & \text { for } j=0
\end{aligned}\right.
$$

Twó remarks are in order here. First, recall that realizations of 0 are not observable. Realizations of $\left\{S_{t}\right\}$ are, but $S\left(\eta_{t}\right)=S\left(\omega_{t}\right)+\nabla\left(\eta_{t}\right)$, where $S\left(\epsilon_{t}\right)=\int S\left(\eta_{t}\right) P\left(d \eta_{t} \psi_{t}\right)$, and $U\left(\eta_{t}\right)=S\left(\eta_{t}\right)-S\left(\omega_{t}\right)$. Since the distribution of $\left[\left(\pi_{t}\right)\right.$ is also determined by $\omega_{t}$, and $\left\{w_{t}\right\}$ is a larkov process, $S_{t}$ is a sur. of two Markov processes. But a process which is a sum of Markov 
processes is not, in general, Markov. So the observable $\left\{S_{t}\right\}$ process is not Markov.

The second point to note concerns the nortality of firms. Issumption (1.iii) insures that there exists a finite $n^{*}$, such that for $n>n^{*}$

$$
\min _{i \in \Omega}\left\{p_{i, 0}^{n}: i \in \Omega\right\} \geq \epsilon>0
$$

where $p_{i, j}^{n}=\operatorname{Pr}\left\{\omega_{t+n}=j \mid \omega_{t}=i\right\}$. Since $p_{0,0}=1$, this inplies that all states but 0 are 'transient'. That is, no matter its initial $\omega$, a firm will, with probability one, reach zero in finite time and stay there. Firms, like people, eventually die.

Since the passive learning model implies that firms can survive forever there is a sense in which this latter result differentiates the model with active exploration from the passive learning model. However, $j$ order to make empirical use of this distinction we would require a very long time series of data. On the other hand, the passive learning model did have the additional implication that the size distribution of survivir firms ought to be stochastically increasing in any finite range of ages (corollary 2.9). For comparison, we now consider the properties of the sequence of survivor distributions generated by the model with active exploration.

Let

$$
Q^{L}=\left\{q \in \mathbb{R}_{+}^{L}: \Sigma q_{i}=1\right\}
$$

be an L-1 dimensional simplex, so that any $q \in Q^{\mathrm{X}+1}$ can be regarded as a 
density on $n$. Note that a potential entrant with an $\boldsymbol{\omega}=0$ would not enter, so that the initial distribution of the $v$ in a cohort is a $p^{0} \in Q^{\mathbb{R}}$. It can be shown (see Ericson and Pakes, 1989) that no atter the initial $p^{0} \in Q^{\mathbb{K}}$, the $w$ and $S$ distributions of the survivors in period $t$, say

$$
P_{w}\left(\cdot \mid t, p^{0}\right) \text { and } P_{S}\left(\cdot \mid t, p^{0}\right)
$$

each converge (point wise), as $\mathrm{t}$ grows large, to a unique invariant distribution, say

$$
\mathrm{P}_{\omega}^{*}(\cdot), \text { and }{ }_{\mathrm{S}}^{*}(\cdot)
$$

(these distributions are invariant to both $\mathrm{p}^{0}$ and to the passage of time). One can actually go one step further than this and show that, given some additional regularity conditions on the location of $p^{0}$ and on the transition probabilities, there vill be a finite $t^{*}$, such that for any $p^{0}$

$$
P_{s}\left(\cdot \mid t+1, p^{0}\right)>_{s w} P_{s}\left(\cdot \mid t, p^{0}\right)
$$

provided $t>t^{*}$. That is, not only does the size-distribution of surviving firms converge to an invariant distribution, but after some ${ }^{*}$ the convergence will be 'monotone' and the size distribution of surviving firms vill stochastically increase from period to period (just as in the passive learning model).

Still, however, the empirical implications of the active learning model on the evolution of the size-distributions of surviving firms are weaker than those of the passive learning model. In particular the active 
learning model does not predict that the size distribution will be stochastically increasing at each age. On the other hand, the active learning model does not bar this event from occurring, and it can predict that the size distribution will be stochastically increasing at later ages.

There is, however, at least one set of observable inplications which differentiate between the two models more sharply. Lecall that in the passive learning model the parameter that deternines the distribution of profits is time invariant. This induces a dependence between the initial size of a firm and the size at any future date. Indeed as equation (2.12) shows, the passive learning model implies the stronger result that the conditional distribution of size at $t$, conditional on the immediate past sizes and the initial size, will always be strictly increasing in the initial size. In the active learning model the parameter determining the firm's profitability distribution, i.e. $u$, evolves over time. Later year size realizations are governed by a different value of $w$ than those from earlier years and, as time passes, the dependence between the later and earlier values of $\omega$, and therefore of size, dies out. This is also true for the conditional distribution of $s_{t}$; i.e. the distribution of $s_{t}$ conditional on immediate past values of $S$ should gradually becone independent of initial year sizes. Yoreover, since the dependence of $w_{t}$ on its history is only through the value of $w_{t-1}$, we aight expect that if ve condition on immediate past sizes the dependence on initial size will die out relatively quickly. Indeed, in the extrene case where $S_{t}=\mathbf{S}\left(w_{t}\right)$, so that sales is a deterministic function of $v_{t}$, the conditional distribution of $S_{t}$ depends only on $S_{t-1}$. In this case a three year panel is enough to differentiate the active from the passive learning model.

When there is noise in the relationship between $w_{t}$ and size we must 
base our distinction between the active and the passive learning model on more detailed properties of the stochastic process generating size conditional on survival. Let $\left\{\mathrm{S}_{\mathrm{t}}^{\mathrm{a}}\right\}_{\mathrm{t}=1}^{\infty}$ be that process (it is described formally in sppendix 2). Then, the active learning model implies that as $T$ grows large the distribution of $\left(\mathrm{S}_{\mathrm{x}+\tau}^{\mathrm{a}}, \mathrm{S}_{\mathrm{x}+\tau+1}^{\mathrm{a}}, \ldots\right)$ becones, roughly spering, independent of realizations of $\left(S_{1}^{a}, \ldots, S_{x}^{a}\right)$. Yore precisely, we have lemma 6 and its implications (explained inmediately after presentation of the lemma).

5. Lemma (-mixing of the $\left\{S_{t}^{a}\right\}$ process).

Let $\left\{\mathrm{S}_{t}^{\mathrm{a}}\right\}_{t=1}^{\mathrm{x}}$ be the stochastic process formed from the distribution of sales conditional on survival and any initial $\omega_{0} \epsilon(1,2, \ldots, \mathbf{K})$, and $\mathbf{y}_{x}^{y}$ be the o-algebra generated by possible realizations of $\mathrm{S}_{\mathrm{x}}^{\mathrm{a}}, \mathrm{S}_{\mathrm{x}+1}^{\mathrm{a}}, \ldots, \mathrm{S}_{\mathrm{y}}^{\mathrm{a}}$. Then $\left\{\mathrm{S}_{t}^{\mathrm{a}}\right\}$ $\phi-$ mixes at a geometric rate, i.e.

$$
\sup \left(\left|P\left(E_{2} \mid E_{1}\right)-P\left(E_{2}\right)\right|, E_{1} \text { with } P\left(E_{1}\right)>0 \text { and } E_{1} \in \mathbf{Y}_{1}^{X}, E_{2} \in \mathbf{Y}_{x+\tau}^{\infty}\right) \leq \Lambda \phi^{\tau}
$$

with $\phi<1$.

Proof. See Appendix 2

Lemma 5 states that any dependence between size realizations that occur after $x+\tau$, and size realizations that occur before $x$, goes down geometrically in $\tau$. It implies that for $k \geq 0$ 
(6) $\sup _{s_{1}}\left[\sum|z| p_{s}\left(z \mid s_{t-1}, \ldots, s_{t-k}, s_{1}, x_{t}=1\right)-p_{s}\left(z \mid s_{t-1}, \ldots, s_{t-k}, x_{t}=1\right) \mid\right] \leq \Delta_{k}$

for some $\phi 1$, on a set of $\left(s_{t-1}, \ldots s_{t-k}\right)$ with probability one. That is b: choosing $k$ sufficiently large we can ake the conditional distribution of $s_{t}$, conditional on $s_{t-1}, \ldots, s_{t-k}$, and $s_{1}$, as close as we like to being independent of the precise realization of $s_{1}$. Note that equation (2.13) insures that this is not the case in the passive learning model. The nex corollary is an immediate implication of (5) and (6).

\section{Corollary}

For any $k \geq 0$

$$
E_{\left(s_{1}\right)}\left|E\left[S_{t} \mid s_{t-1}, \ldots, s_{t-k}, s_{1}, x_{t}=1\right]-E\left[S_{t} \mid s_{t-1}, \ldots, s_{t-k}, x_{t}=1\right]\right| \leq \Delta_{k} \phi^{t}
$$

on a set of $\left(s_{t-1}, \ldots s_{t-k}\right)$ with probability one.

Recall that corollary (2.14) insures that in the passive learning model the conditional expectation of $s_{t}$, conditional on any realization, $\left(s_{t-1}, s_{t-2}, \ldots s_{t-k}, s_{1}\right)$ and survival until $t$, is strictly increasing in $s_{1}$. Hence corollary (1) differentiates the active from the passive learning model. The distinction between the two models is particularly striking, in the special case where $S_{t}=S\left(\psi_{t}\right)$, in which case $\Lambda_{\mathbf{k}}=0$ for $\mathbf{k}>1$

Ve now consider the econometric techniques needed to bring this distinction to data. 


\section{Section 3: Estimation and Testing}

There are two nonparametric implications of the odels we are considering that will be investigated empirically. The first is whether the size distribution of surviving firas is stochastically increasing in age; or whether, for all $\mathrm{t}$

$$
P_{S}(\cdot \mid t) \geq P_{S W} P_{S}(\cdot \mid t-1)
$$

The passive learning model implies it must, while the active exploration model implies it may, but need not - at least in the early ages. The second question posed of the data is whether, for different values of $k$,

$$
E\left[S_{t} \mid s_{t-1}=s_{t-1}, \ldots, S_{t-k}=s_{t-k}, S_{1}=s_{1}, x_{t}=1\right]
$$

is strictly increasing in $s_{1}$. Mgain the passive learning model says it must be. But here there is a sharper contrast with the implications of the active exploration model. The model with active exploration implies that, for $t$ large enough, the regression function in (2) cannot depend on $s_{1}$. To check whether (1) seems consistent with the data, ve will simply plot and compare the size distribution at different ages. It is more difficult to present a pictoral representation of the regression function in (2). Our analysis of its properties must, therefore, be somewhat wore formal.

This section develops an intuitive nonparametric estimator for (2), and then considers tests of whether or not it is increasing in $s_{1}$. Indeed, since both models imply that the regression function is nondecreasing in $s_{1}$, we employ a two-part testing sequence. Ve first test whether (2) is 
weakly increasing in $s_{1}$. If this vere not the case we vould doubt whether either of our models provided an adequate approximation to the process generating the data being analyzed. If, on the other hand, the hypothesis of weak monotonicity is acceptable, ve nove on to test the null of whether the regression function does not depend on $s_{1}$ against the alternative of it being strictly increasing in that variable. Icceptance of both null hypotheses is interpreted as support for the active exploration nodel, while acceptance of only the first is interpreted as support for passive learning.

To obtain our estimator of the regression function ve define $J$ positive numbers, say $\left\{\bar{\sigma}_{j}\right\}_{j=1}^{J}$, and use them to break $\mathbb{R}_{+}$into cells, as in figure 3 . Ve then define the function $\sigma(\cdot): \mathbb{R}_{+} \rightarrow[1, \ldots, J]$ which assigns to each $S_{t}$ the number of the cell it falls into, i.e. for $j=1, \ldots, J$,

$$
\sigma_{t}=\sigma\left(S_{t}\right)=j, \quad \text { if and only if, } \bar{\sigma}_{j-1}<S_{t} \leq \bar{\sigma}_{j} \text {, }
$$

where it is understood that $\bar{\sigma}_{0}=0$, and $\bar{\sigma}_{\mathrm{J}}=\omega$.

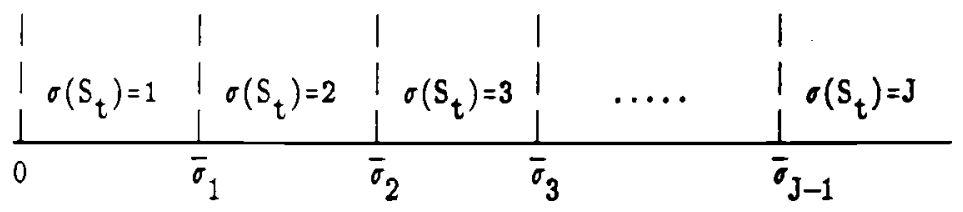

Figure 3: The Punction, $\sigma\left(S_{t}\right)$. 
Similarly for $k<t$ define the function $\sigma^{k}(\cdot): \mathbb{R}_{+}^{k+1} \rightarrow[1, \ldots, J]^{k+1}$, by

$$
\sigma^{k}\left(S^{t-1}\right)=\left\{\sigma\left(S_{t-1}\right), \sigma\left(S_{t-2}\right), \ldots, \sigma\left(S_{t-k}\right), \sigma\left(S_{1}\right)\right\}
$$

In the empirical analysis we treat all values of $S$ that fall into the same cell as equivalent (for the theoretical properties of the test statistics ve require that the cell or 'band' width go to zero at an appropriate rate). For our purposes, then, a $\left\{s_{t-1}, s_{t-2}, \ldots, s_{t-k}, s_{1}\right\}$ history of a firm which survives until period $t$ is one of the $J^{k+1}$ possible values of $\sigma^{k}\left(S^{t-1}\right)$. Each of these values is a $k+1$ dimensional cell, and we denote the set of such cells by $\left\{\sigma_{p}^{k} ; p=1, \ldots, J^{k+1}\right\}$. Our testing procedure is based on estimating the mean and the variance of the regression function in (2) in the intervals defined by these cells.

Yore precisely let $\mu^{k}$ and $\gamma^{k}$ denote the vectors

$$
\mu^{k}=\left[\mu_{p}^{k}=E\left\{S_{t} \mid \sigma^{k}\left(S^{t-1}\right)=\sigma_{p}^{k}\right\}\right]
$$

and

$$
v^{k}=\left[v_{F}^{k}=\operatorname{Var}\left\{S_{t} \mid \sigma^{k}\left(S^{t-1}\right)=\sigma_{p}^{k}\right\}\right] .
$$

Now consider a random sample of firms from the population of interest and let $\mu^{k}$ and $\hat{\gamma}^{k}$ be the sample analogues of $\mu^{k}$ and $\psi^{k}$ (that is the vector of the sample's cell means and the sample's within cell variances). Finally let $\sqrt{1}^{k}$ be the vector containing the square root of the number of firms falling into each cell. Then the central limit theorem and the law of large numbers imply that

$$
\operatorname{diag}\left[\mathbb{N}^{k}\right]\left(\mu^{k}-\mu^{k}\right) \sim N\left(0, \operatorname{diag}\left[V^{k}\right]\right)
$$


while

$$
\operatorname{diag}\left[\hat{V}^{k}\right] \stackrel{P}{\longrightarrow} \operatorname{diag}\left[V^{k}\right]
$$

where diag $[x]$ denotes a diagonal matrix vith $x$ on the principal diagonal, ^) reads converges in distribution, $\stackrel{P}{\longrightarrow}$ denotes convergence in probability, and $N(\cdot, \cdot)$ denotes the nultivariate noral distribution.

Now consider possible values of $\sigma^{*}=\left[\sigma\left(\mathrm{S}_{\mathrm{t}-1}\right), \ldots, \sigma\left(\mathrm{S}_{\mathrm{t}-\mathrm{t}}\right)\right]$. The tes for weak monotonicity of the regression function in $S_{1}$ is a test of whether, for all $\sigma^{*} \in[1, \ldots, \mathrm{J}]^{\mathrm{k}}$

$$
\mu\left(\sigma^{*}, \sigma\left(\mathrm{S}_{1}\right)=\sigma_{1}\right) \geq \mu\left(\sigma^{*}, \sigma\left(\mathrm{S}_{1}\right)=\sigma_{2}\right)
$$

whenever $\sigma_{1} \geq \sigma_{2}$. Similarly the test of whether the realization of $S_{1}$ do not effect the regression function is a test of whether for all $\sigma^{*} \epsilon$ $[1, \ldots, \mathrm{J}]^{\mathbf{k}}$

$$
\mu\left(\sigma^{*}, \sigma\left(S_{1}\right)=\sigma_{1}\right)=\mu\left(\sigma^{*}, \sigma\left(S_{1}\right)=\sigma_{2}\right)
$$

whenever $\sigma_{1} \neq \sigma_{2}$.

Yore formally assume that, for each $\sigma^{*}$, the vector $\mu^{k}$ is ordered by the associated values of $\sigma\left(S_{1}\right)$. Then each of the veak monotonicity constraints can be represented as a linear inequality constraint of the form $I^{\prime} \mu^{k} \geq 0$, when $r^{\prime}=[0, \ldots 0,-1,1,0, \ldots 0]$. Gathering all such

constraints into the matrix $R$, the null hypothesis of weak monotonicity written as 


$$
\mathbf{B}_{\mathbf{Y}}^{0}: \mathbb{R} \mu^{\mathrm{k}} \equiv \mathbf{r} \geq 0
$$

Note that $R$ is of full rov rank, say $C$. Ve vant a test of (6) under the maintained hypothesis that $I \in \mathbb{R}^{\mathrm{C}}$.

Using results dating back to Barlow, Bartholemew, Bremner and Brunk $(19 ; 0)$, Lppendix 3 shows that the difference between an unconstrained estimate of $I$, and an estimate constrained to satisfy (6), cam be used to build a test statistic for the hypothesis in (6), say $\chi_{\mathbf{y}}^{2}$, which, conditional on $r=0$, has a limiting distribution given by a veighted average of chi-square deviates. That is, if we let $\{\boldsymbol{V}(\mathrm{c})\}_{\mathrm{c}=0}^{\mathrm{C}}$ be the required sequence of veights, then the probability that $\chi_{\|}^{2}$ is greater than any a>0, say $\mathrm{T}_{\mathbf{Y}}(\mathrm{a})$, has a limit (as $\mathrm{N}$ or sample size grows large), given by

$$
I_{\mathbf{Y}}(a)=\operatorname{Pr}\left\{x_{\mathbf{M}}^{2}>a \mid r=0\right\}+\underset{c=0}{C} \mathbf{C}(c) \operatorname{Pr}\left\{x_{\mathbf{Y}}^{2}>a\right\}
$$

Note that, if $x_{Y}^{0}$ is the realized value of $\chi_{\mathbf{M}}^{2}, T_{\mathbf{M}}\left[x_{\mathbf{Y}}^{0}\right]$ provides the "p-value" (or the probability of type I error) of a test that would reject the null if $x_{\mathbf{H}}^{2}=x_{\mathbf{M}}^{0}$ when the true value of $r$ was zero. The $p$-value when $I$ is any value greater than zero cannot be larger.

Unfortunately there is no simple vay of calculating the values of the weights, that is of the $\{V(c)\}_{c=0}^{C}$, needed to obtain $T_{\mathbf{Y}}\left[x_{\mathbf{Y}}^{0}\right]$. As a result Appendix 3 develops a simulated estimate of the $\mathbf{V}(c)$, say $\hat{V}^{\prime}=$ $[\hat{V}(1), \ldots, \hat{V}(C)]$, and a consistent (as the number of simulations draws grows large) estimator for the variance-covariance matrix of $\vec{Y}$, say $\vec{V}(\hat{V})$. This allows us to base our empirical work on estimated p-values and their variances. That is, each test result given in the next section will 
contain the two numbers,

(8a)

$$
\hat{\mathrm{T}}_{\mathbf{Y}}\left[x_{\mathbf{M}}^{0}\right]=\sum_{\mathbf{c}=0}^{c} \hat{\mathbf{V}}(\mathrm{c}) \operatorname{Pr}\left\{x_{\mathrm{c}}^{2}>x_{\mathbf{I}}^{0}\right\} \equiv \hat{\mathbf{V}}^{\prime} \mathbf{I} \quad(\text { say }),
$$

and

(8b)

$$
\hat{\mathrm{V}}\left\{\hat{\mathrm{T}}_{\mathbf{Y}}\left[\mathbf{x}_{\mathbf{Y}}^{0}\right]\right\}=\mathbf{I} \cdot \hat{\mathrm{V}}(\hat{\mathbf{V}}) \mathbf{X} .
$$

The test of the null hypothes is that the regression function in (2) does not depend on $s_{1}$ conditional on it being nondecreasing in that variable is a test of the null hypothesis,

$$
\mathrm{H}_{2}^{0}: \quad \mathrm{R} \mu^{\mathrm{k}}=\mathrm{r}=0
$$

under the maintained hypothesis given by $\mathbf{H}_{\mathbf{Y}}^{0}$ in (6). The test statistic for this hypothesis, say $\chi_{Z}^{2}$, is based upon the difference betwet the estimate that satisfies the nonnegativity constraints in (6) and zero: and Appendix 3 shows it to have a limit distribution which, conditional or the null in (9), is also a veighted average of chi-square deviates. Igai: the veights are difficult to calculate but easy enough to simulate. Letting the simulated values of these weights be $\dot{V}=[\dot{V}(1), \ldots, \bar{V}(C)]$, an the observed value of the test statistic be $x_{2}^{0}$, the empirical results for the test that the regression function does not depend on $s_{1}$ conditional 0 it being non-decreasing in that variable each contain the two numbers, 


$$
\hat{\mathrm{I}}_{Z}\left[x_{\mathrm{X}}^{0}\right]=\sum_{\mathrm{c}=0}^{\mathrm{C}} \dot{\mathrm{V}}(\mathrm{c}) \operatorname{Pr}\left\{x_{\mathrm{c}}^{2}>x_{Z}^{0}\right\} \equiv \dot{\mathrm{V}} \mathbf{x}, \quad \text { (say), }
$$

and

$$
\dot{V}\left[\hat{T}_{Z}\left[x_{Z}^{0}\right]\right]=\mathbf{I}, \hat{V}[\hat{V}] \mathbf{x} .
$$

Ve also compare this sequence of tests, that is the test for veak monotonicity under an unconditional naintained hypothesis coupled with the test of the hypothesis that $s_{1}$ has no effect on the regression function conditional on the maintained that any effect is nondecreasing, to the more familiar test of whether $s_{1}$ has no effect on the regression function conditional on an unconstrained maintained hypothesis. The latter test statistic of the null hypothesis in (9), say $\chi_{T}^{2}$, has the familiar chi-square limit distribution with $C$ Degrees of freedom. It can be shown that

$$
x_{T}^{2}=x_{Y}^{2}+x_{Z}^{2}
$$

with probability one. So the observed value for the test of no effect of $s_{1}$ conditional on an unconstrained maintained, say $x_{T}^{0}$, will be just the sum of $x_{Y}^{0}$ and $x_{Z}^{0}$. For comparison, our tables will also provide the p-value of $x_{\mathrm{T}}^{0}, \mathrm{~T}_{\mathrm{T}}\left[x_{\mathrm{T}}^{0}\right]$ (these can be found in standard tables).

Section 5. The Data and the Empirical Results

The data used in this study vere obtained from the Visconsin Department of Industry, Labor and Human Relations' (DILER's) records for unemployment insurance (VI) coverage. The records for the years between 1978 and 1986 (inciusive) were linked together by OI account number by 
David Neuendorf and Ron Shaffer (see Neuendorf and Shaffer, 1987).7

Any private employer hiring at least one vorker and paying at least $\$ 1,500$ in a quarter is required to file information on the number of workers, wages, and OI tax contributions to DILHR. For the purposes of our analysis the first time it does so is treated as the 'birth' of the firm. Size in that, and in subsequent, years is neasured by the number of employees.

The unit used to match observations over time was the DI account number. When a new business changes ownership or legal status, DILHB freezes its current account and either creates a new account, or, in the case of an acquisition, merges the employment information into another account. When this occurs the old account has a successor code, and a ner account, if created, will have a predecessor code. New accounts which were a result of a change in legal status (and therefore had a predecessor code) were separated out and not treated as a part of a birth cohort in this analysis. Analogously we use the successor code to distinguish between attrition due to liquidation, and attrition due to mergers (and other changes in legal status). A major advantage of this type of data is that it can distinguish between these two sources of 'exit'.

Tables 2 and 3 provide information on the evolution of the size distribution of the surviving firms from the 1979 birth cohort in retail and in manufacturing, respectively (recall, from Table 1, that these two sectors account for 80 percent of the employment in our sample). The row labelled 'count' gives the number of firms active in the column age. The row labelled transferring out provides the number of firms which were active in the column year but transferred out (due to a change in legal status) before 1986. This source of attrition accounts for about $8 \%$ of the 
1979 cohort in retail trade, and about $4 \%$ in mufacturing. This should be compared to the extent of liquidation (the figures given in the row labelled mortality rates). Over $60 \%$ of the 1979 birth cohort in retail liquidated before 1986, and the analogous figure in manufacturing was over 50\%. Since liquidation vas quantitatively so much more important a source of attrition in these data, we simply onitted those firms who subsequently changed ownership from the analysis. (Hovever, alnost identical empirical results are obtained if we include the firms in the analysis until the year before they transfer out.)

The passive learning model implies that the proportion of surviving firms with size greater than any $\mathbb{I}$, or the numbers in each row of the body of the tables, should increase with age (i.e., as we move from left to right on the table). We have 'squared off' the adjacent transitions which do not satisfy this condition. On the whole, the consistency of the data with the hypothesis is quite striking - particularly in retail. Of the seventy-seven possible adjacent transitions, only six are decreasing, and none of them indicate a fall of more than 1.0\%. In manufacturing there are nine transitions which decrease; two fall by more than $1.5 \%$, and two more by $.6 \%$. Given the possibilities for reporting and recording errors in this type of data (see Neuendorf and Shaffer, 1987), if the null vere true, we would not find these results to be 'surprising'. That is, to us these results are quite consistent with the implications of passive learning indeed amazingly so for retail trade. Note also that, in both sectors, the means are strictly increasing in age.

$A$ more detailed look at these two tables uncovers some revealing contrasts between the evolution of the size distribution in the two sectors. The size distribution in the initial year is not much different 
between the two sectors; indeed if anything the initial size distribution is slightly 'larger' in retail trade (retail has the larger initial year mean, 5.4 vs. 4.9 , and a higher percentage of the firns in the largest size classes). Hovever, by age eight this ordering has turned around. That is, by age eight the size distribution for manufacturing is stochastically larger (even in the strict sense) than that in retail (the means are 13.3 vs 8.8 , and manufacturing has over twice the fraction of firms with 50 or more employees). The size distribution is stochastically increasing in age in both sectors, but it is increasing at a much more rapid rate in manufacturing.

Yoreover, the age eight distribution in retail is quite close to the cross-sectional distribution of all retail firms active in 1978 (or 1986, see the last two columns of the table). Both have about $3 \%$ of their firms with more than 50 employees (though the cross-sectional distribution still has the larger mean, 14 vs. 9). In contrast, the age eight distribution in manufacturing is much smaller than the 1978 cross-sectional distribution in that sector. In manufacturing the cross-sectional distribution has more than three times the fraction of firms with more than 50 employees (19.6 vs. 6.5), and a mean wich is almost six times that from the age eight distribution (73.8 vs. 13.3). Thus, if ve vere to think of the cross-sectional distribution as an approxination to the limit distribution (even though formally it is not), then we night conclude that by age eight the retail cohort had almost reached $i t$, but the munacturing cohort was still nowhere near its limit distribution. Indeed, if we also assuned that eight years was enough time to form a fairly precise posterior about a time invariant profitability paramenter, then we vould conclude that the data from retail was supportive of the passive learning model, but the data from 
manufacturing was not.

A more formal check of the consistency of the data with the two models can be derived from an analysis of the regression for size at age eight on size in the immediate preceding periods, and size at age one. Both nodels imply that this function will be veakly increasing in initial size, but the pass :e learning model implies that it be strictly increasing in that variable, and the active learning model inplies that it will not.

Tables 4 and 5 provide some evidence on the relevant hypothesis. Because there were less than half the number of entering firms annually in manufacturing, ve aggregated the 1979 and 1980 manufacturing cohorts and examined the regression for expected sales at age seven of the aggregated cohort. The cell size cutoffs vere set at the beginning of the analysis and not changed thereafter. For the veak monotonicity, and the zero conditional on monotonicity, restrictions, we have presented tro sets of 'p-values' for each observed value of the test statistic. The first column provides the simulated estimates of the true p-values as explained in Section 4 (the estimated standard errors of these estimates appear in parentheses below their values). The second column provides the p-values that vould be obtained if the components of the estimator of the vector of constraints being tested had mutually independent distributions under the null. In this case the veights required for the calculation of the limit distribution (see equation 4.7) have an analytic form (see appendix 3 ), so there is no need for simulation. Though the independence assumption is wrong in our (and probably in most) cases, it does provide an easily calculable approximation to the non-analytic true $p$-value which night be of use in (at least) the preliminary stages of analysis if the approximation produced numbers that vere sufficiently close to those ve are after. 
Comparing columns (1) and (2) in the next four tables it is clear that in the cases where the true p-values were low (say less than .10), so that there was some chance of rejecting the null, the approximation did produce a value within .05 of the value we vere after.

Note first that none of the tests reject veak nonotonicity at traditional levels of significance. So both the retail and the manufacturing data are consistent with the hypothesis that the regression function is nondecreasing in $s_{1}$, just as both our vodels predict. There the similarity in the test results on the two data sets ends. In retail it is clear that if we condition on one lagged value of $S$, that is on realizations of $S_{7}$, and then vary $s_{1}$, firms with larger $s_{1}$ have larger average sales at age 8 . There is really no doubt about this point as the p-value of the test statistic is essentially zero, so we would reject the null at any traditional significance level. The same is true if we condition on $s_{7}$ and $s_{6}$; or on $s_{7}, s_{6}$ and $s_{5}$; or even on $s_{7}, s_{6}, s_{5}$ and $s_{4}$; and then vary $s_{1}$. In all these cases realizations of $s_{1}$ have an independent effect on the expectation of sales at age eight. This dependence only starts to become insignificant at five percent significance levels when ve condition on five past sales realizations. Hovever, this might vell be a result of the possibility that, with our limited amount of data, a fifth order nonparametric autoregression would provide an adequate approximation to the expectation for size generated from any stochastic process - (-mixing or not; we come back to this point below).8

The results for the test of zero conditional on veak monotonicity are strikingly different in manufacturing. Table 5 indicates that, in manufacturing, once ve condition on a single lagged value of $S$, i.e. a realization of $S_{6}$, any differences in $s_{1}$ do not effect the expected size at 
age seven. This time there is little doubt about accepting the null as the p-value is well above .5. Yoreover, the same results obtain if we condition instead on $s_{6}$ and $s_{5}$; or on $s_{6}, s_{5}$, and $s_{4}$; or $s_{6}, s_{5}, s_{4}$ and $s_{3}$.

Tables 6 and 7 push the nonparametric analysis one step further and ask what order of larkov process provides an adequate nonparanetric fit to the (axpectation from the) stochastic process generating size conditional on survival in retail and in manufacturing. The tests in these tables follow a pattern analogous to that in Tables 4 and 5 . That is, ve first test whether first year size, size in the first two years, ..., have a nondecreasing effect conditional on the variables left in the regression function; and then test whether we can accept a zero effect conditional on any of the existing effects being nondecreasing. Igain the results are quite clear. Ve never reject weak monotonicity. In retail we need a fifth order nonparametric Iarkov process to adequately approximate the data. Recall that this is precisely the same ' $k$ ' we needed before we could accept the null that the conditional regression function for size, conditional on $s_{t-1}, \ldots, s_{t-k}$, did not depend on $s_{1}$. In contrast, in manufacturing a third order nonparametric Yarkov process seems to provide an adequate fit to the data. That is, in manufacturing there is a distinction between the orders needed for the -mixing and the larkov tests (compare tables 7 and 5 ). Table 5 says that conditional on realizations of $S_{6}$ realizations of $S_{1}$ do not affect the regression function. Table 7 says that realizations of $S_{5}$, and of $S_{4}$, do. The active exploration model explains this difference by allowing the parameter that determines the size distribution to evolve over time in a 'smooth' fashion, so that its value in year 5 vill tend to be closer to its value in year 7 , and therefore have a more distinct effect on 
the regression function for $S_{7}$, than its value in year 1 will.o

Section 6. Further Implications of the Empirical Results.

Our empirical results can be summarized quite succinctly. The nonparametric implications of the active exploration nodel are consistent with the data in manufacturing, while the nonparanetric inplications of the passive learning model definitely are not. On the other hand, the nonparametric implications of the passive learning nodel seem consistent with the data in retail trade, while those from the active learning model do not. These distinctions ought to effect the type of models we use to analyze phenomena that depend upon firm-specific uncertainties and differences in output paths among firms within an industry; phenomena such as the behavoir of capital markets when there are significant failure probabilities, or supply responses to environmental and policy changes that can induce exit.

The nonparametric results ought also to effect how we account for liquidation induced attrition in the analysis of longitudinal firm-level data. As an example of the importance of such corrections, consider the following excerpt from Davis, Gallman, and Jutchins, "Productivity in American Whaling: The New Bedford Fleet in the Nineteenth Century."

"The age of the vessel (entered as age and age squared) also captures the effects of more than a single set of factors. Elements of vear and tear that influenced productivity, a technical characteristic that one might hope to capture in the age variable, are confounded with the consequence of qualitative differences among survivors; ineffective vessels vere transferred 
by their owners to other activities, were condemned at an early age, or were destroyed in service."

Davis, Gallaan and Eutchins (1987) p.26.

The quotation illustrates how even one of the nost traditional of varioles (age), in one of the most traditional of settings (productivity analysis), can have its "structural" effects (as a measure of the likely extent of physical deterioration) confounded by the self-selection process induced by the endogeneity of the liquidation decision (it also demonstrates a remarkable understanding of the environment generating the data). Davis, Gallman and Butchins (1987) do indeed find a significant positive first order effect of age on vessel productivity.

To see how the nonparametric implications used to test for the relevance of alternative models can also be used to separate out the structural production function coefficients in examples such as this one, assume that output is a parametric function of inputs, say $f\left(x_{t}, \beta\right)$, and an additive disturbance, say $\epsilon_{t}$, whose value is not known when input decisions are made. Then the expectation of output $\left(y_{t}\right)$, conditional on the current value of inputs $\left(x_{t}\right)$, survival until period $t\left(x_{t}=1\right)$, and the information set available in $t-1\left(J_{t-1}\right)$ is a sum of two functions; the structural production function, and the expectation of the disturbance conditional on $x_{t}=1$ and $\mathrm{J}_{\mathrm{t}-1}$, i.e.

$$
E\left[y_{t} \mid x_{t}, x_{t}=1, J_{t-1}\right]=f\left(x_{t}, \beta\right)+E\left[\epsilon_{t} \mid x_{t}=1, J_{t-1}\right]
$$

Now note that both models imply that the decision 8 s to whether to operate the $f$ irm in year $t$ is determined by information available in $t-1$ (i.e. $x_{t}$ 
is measurable with respect to $\left.\mathrm{J}_{t-1}\right)$, so the last term depends only on variables in $J_{t-1}$. This implies that none of the deterninants of $E\left[y_{t} \mid x_{t}\right.$, $\left.\chi_{t}, J_{t-1}\right]$ are determinants of both, $f(\cdot ; \beta)$ and $B\left[\epsilon_{t} \mid \chi_{t}=1, J_{t-1}\right] \cdot$ is a result, once ve determine which of the dynanic nodels are relevant for the data at hand, and therefore what variables deternine $E\left[\epsilon_{t} \mid x_{t}=1, J_{t-1}\right]$, we can, under mild regularity conditions, obtsin a (root $n$ ) consistent asymptotically normal estimator for $\beta_{0}$ (the true value of $\beta$ ) by nininizing a distance between $y_{t}$ and the sum of $f\left(x_{t}, \beta\right)$ and a nonparanetric estinator for the 'nuisance' function $E\left[\epsilon_{t} \mid \chi_{t-1}=1, J_{t-1}\right]$ (for detsils see Robinson, 1988). Note that this method of correcting for the selection process induced by liquidation behavior is fully consistent with the economic models generating liquidation behavior and does not either; 1) depend on the precise functional form of the relevant dynanic stochastic processes; or 2) require a solution to the computationally difficult problem of finding the optimal stopping states as a function of the parameters of the nodel.

If the model with active exploration vere relevant then the distribution of $\epsilon_{t}$ conditional on $J_{t-1}$ would be determined by productivity realizations in the immediately proceeding periods and the anount of exploratory investment; while, in the passive learning nodel, this expectation would depend on age and earlier, as vell as the inediately preceeding, productivity realizations. So the selection correction procedure would differ with the nonparametric implications of the behavioral model assumed to generate liquidation decisions: implications which can be checked for their consistency with the data using the framework outlined above. 


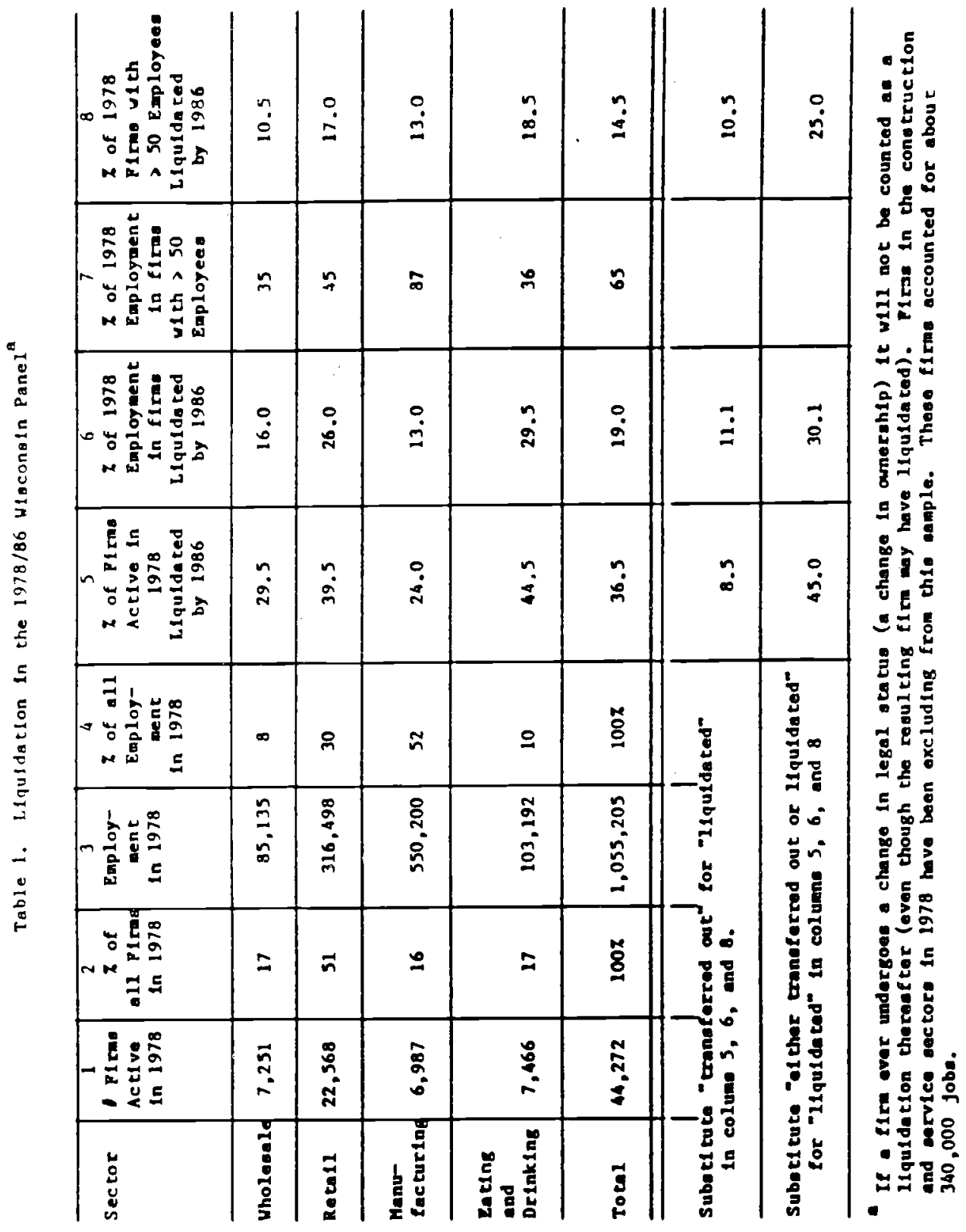



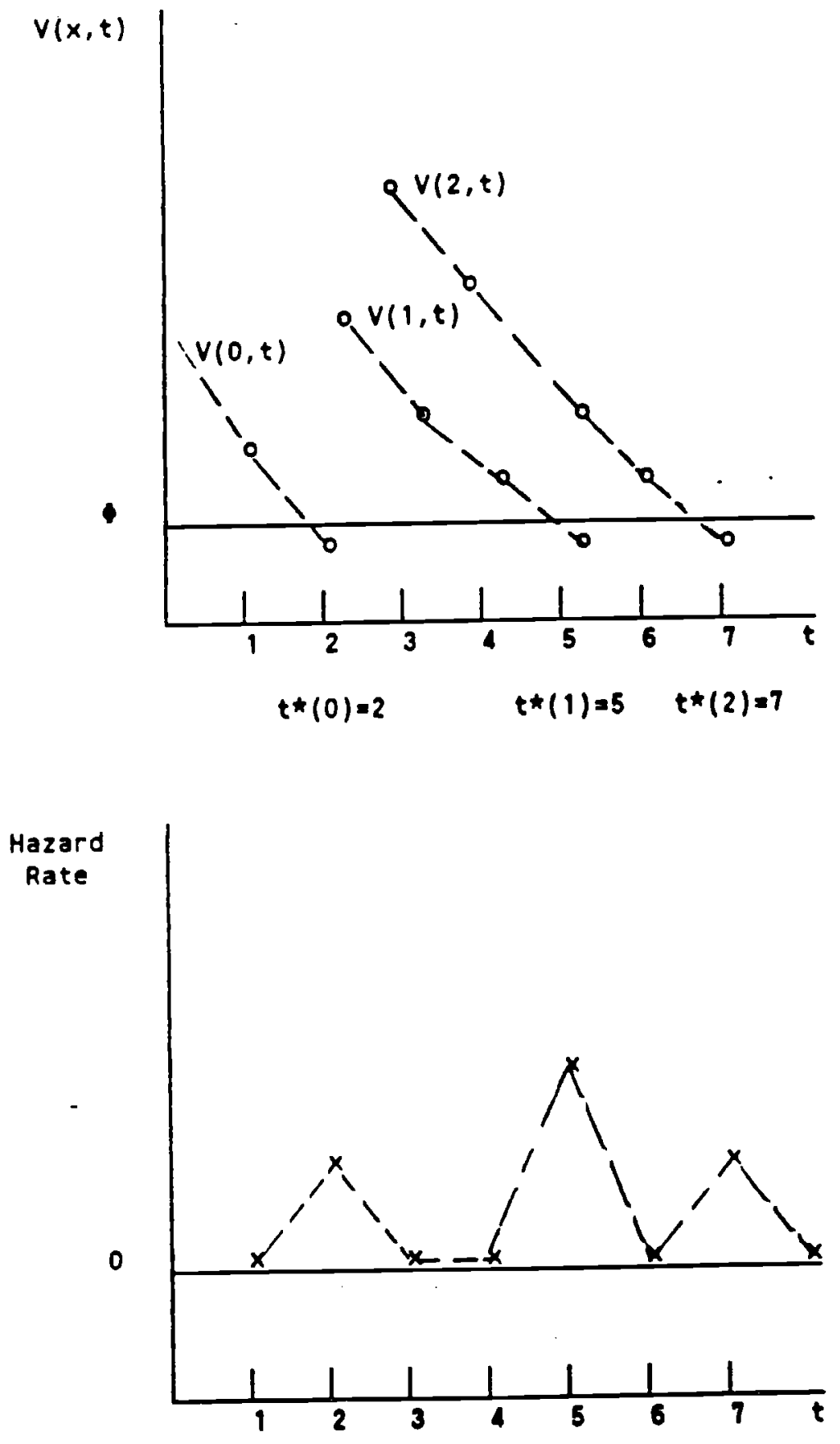

Figure 1: A Beta/Binomial Example 


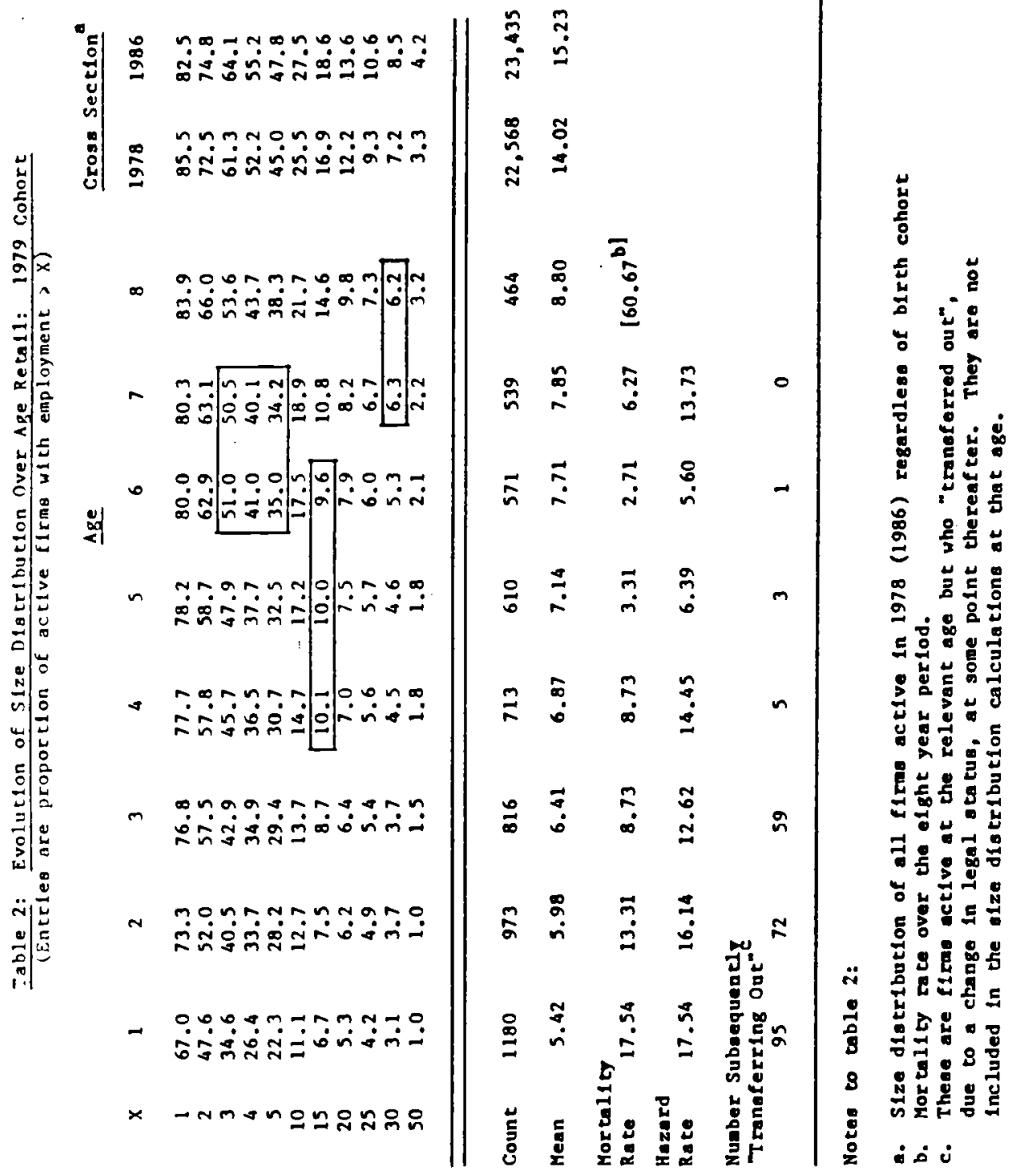




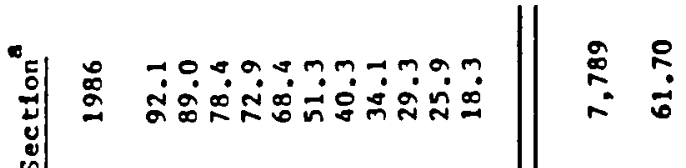

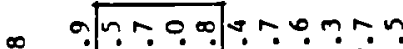

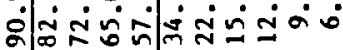
of

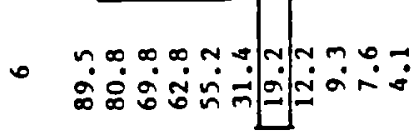

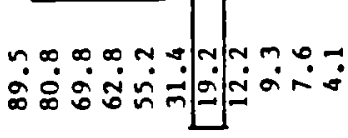

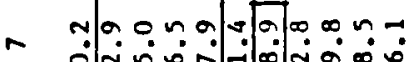

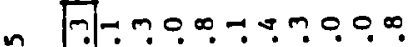
மீi் in

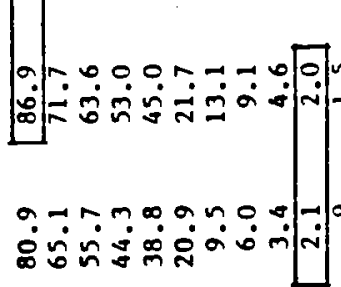

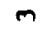

苛

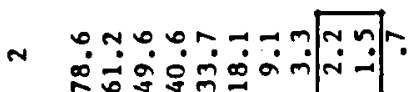

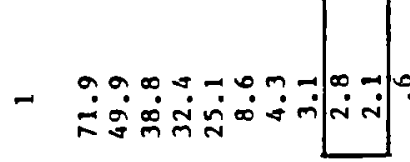

× -Nm \&ீர்

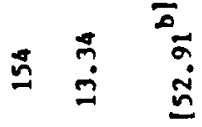

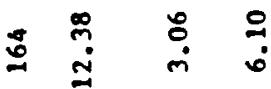

ก กั

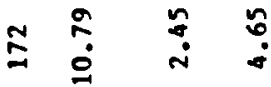

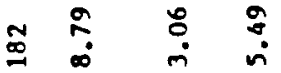

-

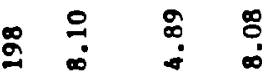

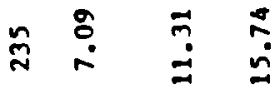

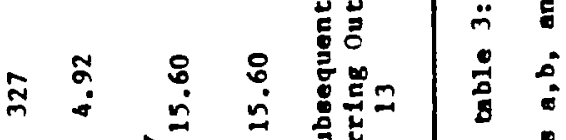

0
0
0
0
0
0
0
0
0
0
5

8

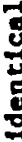

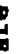

i

g

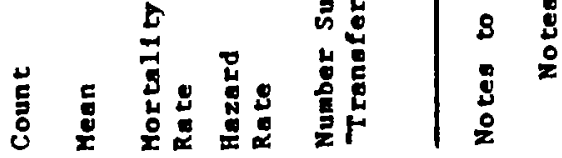


Table 4. Tests for Mean Independence of the Diatribution of $s_{t}$

Conditional on $s_{t-1}, \ldots, s_{t k}$, from $s_{1}$

Data: Reta11, 1979 Cohort and t-8,

S1ze Ciltollg: $2,3,10,25,30$, tw

\begin{tabular}{|c|c|c|c|c|c|c|c|c|c|c|c|}
\hline k & $c$ & $\begin{array}{c}\text { Neak } \\
\text { Monotonlcl ty }\end{array}$ & $\begin{array}{l}c 1 t y \\
p-v a l u \\
(1)\end{array}$ & & $\begin{array}{c}\text { Zer } \\
\text { on } \\
\text { C }\end{array}$ & $\begin{array}{l}\text { Zero Cond1tional } \\
\text { on Montonlet ty }\end{array}$ & $\begin{array}{l}\text { lonal } \\
1 \text { ty } \\
\text { p-val } \\
(1)\end{array}$ & $\begin{array}{l}\text { ues }^{b} \\
(2)\end{array}$ & $\begin{array}{l}\mathrm{Ot} \\
\mathrm{DF}\end{array}$ & $\begin{array}{c}\text { ond } 1 \text { t } \\
\text { zero } \\
x_{T}^{0}\end{array}$ & p-value \\
\hline 1 & 17 & 1.1 & $\begin{array}{l}1.00 \\
(.00)\end{array}$ & .99 & 17 & 37.2 & $(.00)$ & 0 & 17 & 38.2 & .00 \\
\hline 2 & 22 & 6.5 & $\begin{array}{l}.88 \\
(.03)\end{array}$ & .80 & 22 & 23.9 & $\begin{array}{l}.00 \\
(.00)\end{array}$ & .02 & 17 & 30.4 & .11 \\
\hline 3 & 25 & 11.5 & $\begin{array}{l}.66 \\
(.05)\end{array}$ & .52 & 25 & 28.0 & $\begin{array}{l}.00 \\
(.00)\end{array}$ & .01 & 25 & 39.5 & .03 \\
\hline 4 & 22 & 19.1 & $\begin{array}{l}.05 \\
(.01)\end{array}$ & .05 & 22 & 19.1 & $\begin{array}{l}.04 \\
(.01)\end{array}$ & .08 & 22 & 38.2 & .02 \\
\hline 5 & 19 & 17.6 & $\begin{array}{l}.05 \\
(.01)\end{array}$ & .07 & 19 & 13.6 & $\begin{array}{c}.12 \\
(.02)\end{array}$ & .19 & 19. & 31.2 & .04 \\
\hline
\end{tabular}

cohort dimensions: number in cohort $-1,275$; number of flrms reachlng age elght $=464$.

The value in coluan (1) is a olmulated estiate of the true pralue and the value fust below $1 t$ is the etandard error of this estiate. Ten - Irulation draws vere used to calculate the eatinates of the orthant probabllities. The value in column (2) lo obtained by assuning each orthant has equal probability (sce the explanation in the taxt). 
Table 5. Tests for Mean Independence of the Diatribution of $s_{t}$ Conditional on $s_{t-1}, \ldots, s_{t}, k^{\prime}$ froa $s_{1}$

Data: Menufacturing, Combined 1979 and 1980 Cohorts for $t=7.8$

Size Cutoffs: $\quad 2,5,10,25,50,+\cdots$

\begin{tabular}{|c|c|c|c|c|c|c|c|c|c|c|c|}
\hline \multirow[t]{2}{*}{$k$} & \multicolumn{3}{|c|}{$\begin{array}{l}\text { Heak } \\
\text { Monotogle1ty }\end{array}$} & & \multicolumn{3}{|c|}{ Zero Conditional } & & \multicolumn{3}{|c|}{$\begin{array}{c}\text { Uncond 1 t lonel } \\
\text { Z ro }\end{array}$} \\
\hline & C & $x_{H}$ & $\begin{array}{l}\text { p-va } \\
(1)\end{array}$ & $\begin{array}{l}\text { lues } \\
\text { (2) }\end{array}$ & $c$ & $x_{2}$ & $\begin{array}{l}\text { prva } \\
(1)\end{array}$ & $\begin{array}{l}\text { ues }^{0} \\
(2)\end{array}$ & Df & $x_{T}$ & p-value ${ }^{0}$ \\
\hline 1 & 16 & 8.0 & $\begin{array}{l}.54 \\
(.06)\end{array}$ & .44 & 16 & 3.5 & $\begin{array}{l}.57 \\
(.07)\end{array}$ & .86 & 16 & 11.5 & .78 \\
\hline 2 & 25 & 17.6 & $\begin{array}{l}.19 \\
(.03)\end{array}$ & .17 & 25 & 5.8 & $\begin{array}{l}.79 \\
(.03)\end{array}$ & .91 & 25 & 23.6 & .55 \\
\hline 3 & 23 & 14.3 & $\begin{array}{l}.28 \\
(.05)\end{array}$ & .27 & 23 & 4.9 & $\begin{array}{l}.81 \\
(.06)\end{array}$ & .92 & 23 & 19.3 & .67 \\
\hline 4 & 15 & 10.1 & $\begin{array}{l}.13 \\
(.02)\end{array}$ & .24 & 15 & 5.9 & $\begin{array}{l}.54 \\
(.03)\end{array}$ & .59 & 15 & 16.0 & .39 \\
\hline
\end{tabular}

${ }^{8}$ Firm dimensions: number born in cohorts $=737$, number of firms reaching age seven $=353$.

${ }^{b}$ see note b to Table 4 . 
Table 6. Markov Tests for Properties of Retall Regression Punction for Slze at Age Elght

Data: Reta11, 1979 Cohort

S1ze Cutoffs: $2,5,10,25,50,+e^{b}$

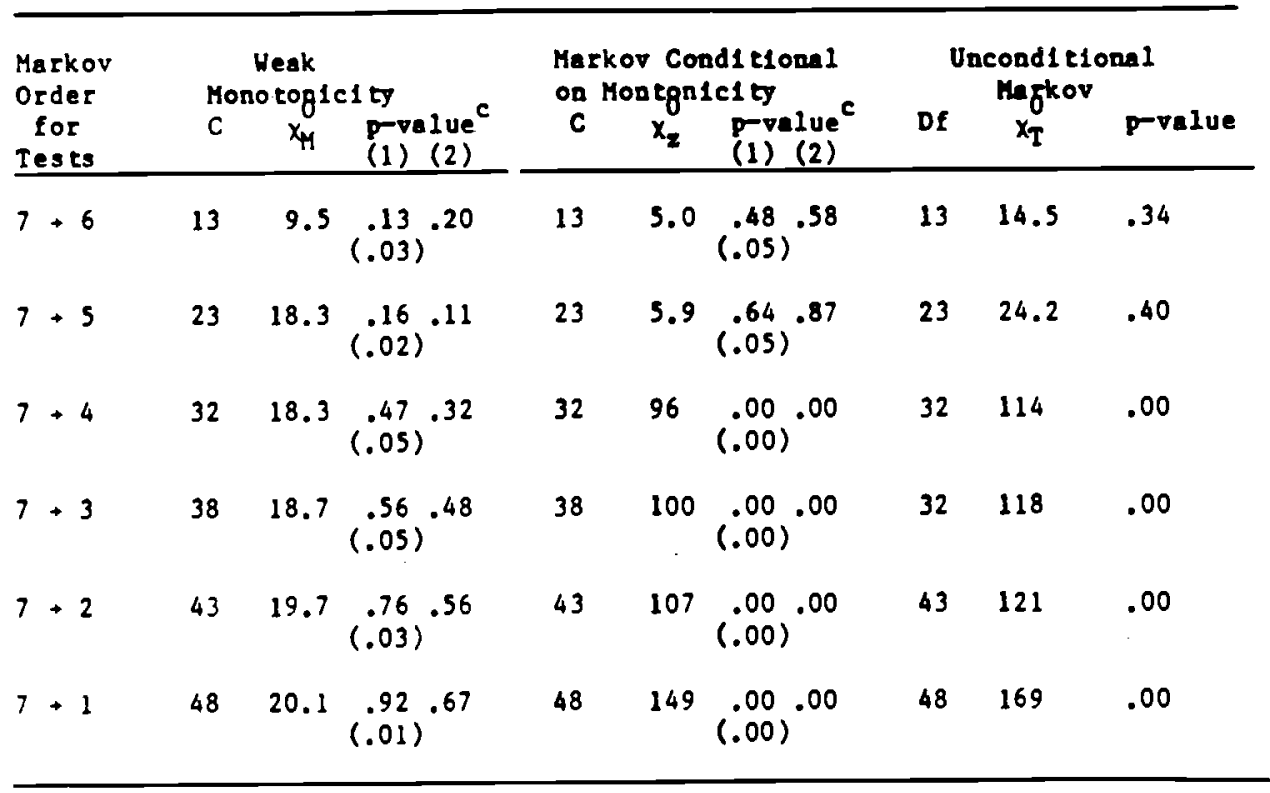

${ }^{a}$ Cohort Dimensions: number in cohort - 1275; number of firms reaching age eight $=465$; number in cells with $>2=291$.

${ }^{b}$ Cell Dinensions: possible number $=279,936$; number populated 228; number w1th $>2$ observations $=54$.

csee note b, Table 4 . 
Table 7. Tests for Properties of Manufacturing Regression Function for Size at Age Seven

Dntn: Mnnifncturing, Combined 1979 and 1980 Cohorts"

Size Cutoffs: $2,5,10,25,50,+\infty^{b}$

\begin{tabular}{|c|c|c|c|c|c|c|c|c|c|}
\hline \multirow{2}{*}{$\begin{array}{l}\text { Markov } \\
\text { Order } \\
\text { for } \\
\text { Teats } \\
\end{array}$} & \multicolumn{3}{|c|}{$\begin{array}{l}\text { Veak } \\
\text { Monotopicity }\end{array}$} & \multicolumn{3}{|c|}{$\begin{array}{l}\text { Markov Conditional } \\
\text { on Montgnicl ty }\end{array}$} & \multicolumn{3}{|c|}{$\begin{array}{l}\text { Uncondi tional } \\
\text { Magkov }\end{array}$} \\
\hline & C & $x_{y}$ & $\begin{array}{l}\text { p-value } \\
\text { (1) (2) }\end{array}$ & C & $x_{2}$ & $\begin{array}{l}\text { p-value c } \\
(1)(2)\end{array}$ & DE & $x_{\tau}^{0}$ & p-value \\
\hline $6+5$ & 9 & 11.9 & $(.02)^{.04}$ & 9 & 2.0 & $(.65 . .75$ & 9 & 14.0 & .12 \\
\hline $6+4$ & 15 & 13.3 & $(.09)^{.09}$ & 15 & 11.7 & $(.07 . .16$ & 15 & 25.1 & .05 \\
\hline $6+3$ & 25 & 15.5 & $(.05)^{.24}$ & 25 & 17.6 & $(.11 .17$ & 25 & 33.1 & .13 \\
\hline $6+2$ & 31 & 16.1 & $(.04)^{.42}$ & 31 & 61.3 & $(.00)^{.00}$ & 31 & 77.4 & .00 \\
\hline $6+1$ & 37 & 16.3 & $(.04)^{.66}$ & 37 & 76.0 & $(.00)^{.00}$ & 37 & 92.3 & .00 \\
\hline
\end{tabular}

cohort Dlmenslons: number of flrms - 737; number of flrms reachlog age seven = 353; number in cells with $>2=179$.

${ }^{b}$ Cell Dimensions: possible number $=46,656$; aumber populated 217 ; number with $>2$ observations 43 .

csee note b, Table 4 . 


\section{rootnotes}

1 See Beckman and Robb, 1985, and the literature cited there, for a discussion of related issues in static framework.

\section{More detailed discussions of $\ell r$ orderings can be found in Milgron} (1981), Ross (1983), and Iarshall and Olkin (1979, chapter 18).

3 Two points should be noted here. First we are ignoring the effect (on both $x(\cdot)$ and $S(\cdot))$ of random variables which have the sme value for different individuals at the same point in tine, but differ in value over time (this vould have occurred in our exaple if prices had varied over time). It the cost of complicating the notation ve could add a price process to our problem without changing any of our ajor results (though some modifications would have to be made to the procedure that matches the model to data; see below). Second, it should be noted that the interpretation of $x(\cdot)$ and $S(\cdot)$ as mappings from realizations of $\eta$, would only be appropriate for our example if $\eta$ vere realized before input decisions were made (Iarscnak and Andrews, 1944). In this case both output and inputs can be determined from $\eta_{t}$, and the size measure can be either output produced or inputs purchased. The extreme alternative is to assume there is no within-period adjustment to $\eta$ (Zellner, Imenta, and Dreze, $1966)$, in which case inputs are chosen to maxime $a_{t+1} \mathbf{E}_{(t)} \eta_{t+1} \mathbf{P}\left(\ell_{t+1}\right)-$ $k_{t} \ell_{t+1}$, where $E_{t}$ provides expectations conditional on current information (and will be defined more precisely below). In this case $\pi(\cdot)$ and $S(\cdot)$ would be interpreted as mappings from $E_{t} \eta_{t+1}$ to $E_{t}{ }_{t+1}$, and input demand in period $t+1$ respectively. There are, of course, intermediate cases where within period adjustment is either partial, or more costly (the appropriate characterization is likely to depend upon the characteristics of the industry being studied). Ne shall come back to some of the alternatives belok, but for now suffice it to note that the results ve focus attention on do not depend on the timing of the input decision.

4 The following counterexample shows that this vould not be the case if we were to assume only a veaker first order stochastic dominance ordering. Let $\theta=\left(\theta_{1}, \theta_{2}\right)$ with $\theta_{2}>\theta_{1}$, and consider the following family of densities (with respect to the counting neasure): $p\left(\eta=2 \mid \theta_{2}\right)=$ $\mathrm{p}\left(\eta=4 \mid \theta_{2}\right)=1 / 2$, and $\mathrm{p}\left(\eta=1 \mid \theta_{1}\right)=\mathrm{p}\left(\eta=3 \mid \theta_{1}\right)=1 / 2$. Clearly, $\mathrm{P}_{\eta}\left(\cdot \mid \theta_{2}\right)$ $>_{s} P_{\eta}\left(\cdot \mid \theta_{1}\right)$. Hovever, if $\eta_{1}=2$, the posterior is $\theta=\theta_{2}$ with probability one, whereas if $\eta_{1}=3$, the posterior is $\theta=\theta_{1}$ with probability one; i.e., the posterior for $\eta=2$ dominates the posterior for $\eta=3$.

5 The assumptions that is the same known value for all agents, and is constant over time, are made for expositional convenience. What is required is that not increase too rapidly with $n^{t}$. Iore precisely, if 
$V_{t}\left(n^{t}\right)$ is the value of continuing in operation at $t$ given that $\eta^{t}=n^{t}$ (a more precise definition of this function is given belov), then what we need is that $v_{t}\left(n^{t}\right)-t_{t}\left(n^{t}\right)$ be nondecreasing in $n^{t}$. Of course, the actual behavior of "exit values" is an eapirical question. If the process generating the exit ve are modelling is indeed a liquidation process, and not a process generated by changes of ovnership and continued operation of the firm in a different guise, the assunptions ve require ought not to be problematic.

- Just as in our description of the passive learning nodel ve vill assume here, for expositional simplicity, that input choices are made after the realization of $\eta$, that liquidation values are a constant and that there is no time-specific, firm-invariant process. Further, the formulation presented here assumes that the conditional distribution of $\tau$ does not depend on $\psi$, an assumption not required for our results.

7 Ve are grateful to them for granting us access to their data, and for graciously answering our subsequent queries. More detail on the data can be found in the appendix of Nevendorf and Shaffer (1987). Though

multiestablishment firms have a choice as to whether to report as a single, or as miltiple units, the establishments of multiestablishment firms that reported separately have been merged into single observations. This should therefore be thought of as firm-level data.

8 Ve have been motivating our two-part testing sequence as a vay of providing additional information on the relevance of alternative models. Inequality tests vere originally motivated as providing more powerful ways of testing a given null. Table 4 also illustrates this point. Take, for example, the case where $k=2$. The $p$-value in column 2 for acceptance of the null that realizations of $S_{1}$ do not matter under the maintained hypothes is that any effect of $S_{1}$ is non-decreasing, is zero; but the p-value for the test that $S_{1}$ does not matter under the unconstrained maintained hypothes is (the unconditional zero columns) is a traditionally acceptable .11.

- Footnote 2 discussed the possibility that input decisions are either wholly, or partially, made before the realization of $\eta$, and concluded by asserting that the various alternatives vould not affect the results ve focus on. Table 7 , insures this is so for the very special, but important, case which Jovanovic's (1982) original article was based on. His assumptions vere a special case of the following ones; the process generating $\left\{\eta_{t}\right\}$ conditional on $\theta$ was i.i.d., the posterior for $\theta$ had sufficient statistics $\left(x_{t}, t\right)$ with $x_{t}=f_{t}\left(x_{t-1}, \eta_{t}\right)$ for sone $f_{t}(\cdot)$, and that no input could be adjusted after any information about $\eta_{t}$ was available. In this case, if input quantities vere our size measure, size in period $t$ is determined by $\left(x_{t-1}, t\right)$ and for a given $t$, there is a $1: 1$ correspondence between $S_{t-1}$ and $x_{t-2}$. So size is a first order larkov 
process. This conclusion vould be destroyed if some, say costly, adjustments could be made after $\eta$ vere realized, or if there vere any dependence in the process generating $\left\{\eta_{t}\right\}$ conditional on $\theta$. Iowever, if Jovanovic's restrictions vere true, the passive learning aodel would satisfy the constraint that the regression for $S_{t}$ conditional on $S_{t-1}, \ldots$, $S_{t-k}$ does not depend on $S_{1}$ provided $k \geq 1$; i.e., it would satisfy the constraint used to test for the active learning model. On the other hand Table 7 makes it clear that the stochastic process generating size is not first order larkov, so the special case discussed by Jovanovic (1982) is not relevant. 


\section{References}

Barlow, B.E., D.J. Bartholemew, J.I. Bremer, L B.D. Brunk (1972); Statistical Inference Onder Order Restrictions: The Theory and Spplication of Isotonic Regression, New York; John Viley $t$ Sons.

Billingsley, P. (1968); Convergence of Probability Ieasures, New York; John Viley Sons.

Billingsley, P. (1979); Probability and Yeasure, New York; John Viley \& Sons.

Blackwell, D. (1965); "Discounted Dynamic Programing," Annals of Yathematical Statistics, Vol. 36, 1965, pp. 226-35.

Chamberlain, G. (1985); "Beterogeneity, Omitted Variable Bias, and Duration Dependence," in J. Heckman and B. Singer (ed.); Longitudinal Analys is of Labor Market Data, Cambridge Oniversity Press.

Churchi11, B. (1955); "Age and Life Expectancy of Business Firms," Survey of Current Business, Vol. 25, pp. 15-20.

Davis, L., B. Gallman, T. Hutchins (1987); "Productivity in Inerican Whaling: The New Bedford Fleet in the Nineteenth Century, "Department of Economics, the California Institute of Technology. 
Dunne, T., Y. Roberts, L. Samuelson (1987); "Plant Failure and Employment Growth in the O.S. Manufacturing Sector," Department of Economics, The Pennsylvania State Oniversity.

Dynkin, E.B. and A. A. Yushkevich (1975); Controlled Yarkov Processes and Their Applications, Nauka, loscow. (English translation published by Springer-Verlag, New York (1979)).

Ericson, R., A. Pakes (Revised 1989); "An Alternative Theory of Firm and Industry Dynamics," Department of Econonics, Columbia University.

Evans, D. (1987a); "The Relationship Between Firm Growth, Size, and Age:

Estimates for 100 Manufacturing Industries," Journal of Industrial Economics, Yol. 35 , pp. .

Evans, D. (1987b); "Tests of Alternative Theories of Firm Growth, " Journal of Political Economy, Vol. 95, pp. 657-674.

Goldberger, A. (1987); "One-sided and Inequality Tests for a Pair of Means," Social Systems Research Institute (No. 8629), Oniversity of Visconsin.

Gourieroux, C., A. Bolly, A. Monfort (1982); "Likelihood Ratio Test, Vald Test, and Ruhn-Tucker Test in Linear Models with Inequality Constraints," Econometrica, Vol. 50, pp. 63-80. 
Heckman, J. (1981); "Statistical Yodels for Discrete Panel Data," in C.

Manski, \& D. McFadden (ed.); Structural Analysis of Discrete Data with Econometric Applications, pp. 114-178.

Heckman, J., \& B. Singer (1985); "Social Science Duration Analysis," in J. Heckman, and B. Singer (ed); Lonzitudinal Inalysis of Labor larket Data, Cambridge Oniversity Press.

Heckman, J., and R. Robb (1985); "Mlternative Methods for Evaluating the Impact of Interventions," in J. Ieckman and B. Singer (ed.), Longitudinal Analysis of Labor Yarket Data, Cambridge Oniversity Press, 1985.

Jovanovic, B. (1982); "Selection and the Evolution of Industry," Econometrica, 50, pp. 649-70.

Lippman, S., \& R. Rumelt (1982); "Uncertain Immitability: In Inalysis of Interfirm Differences in Efficiency Onder Competition," Bell Journal of Economics, Vol. 13, pp. 418-438.

Marschak, J., \& W. H. Andrews Jr. (1944); "Random Simultaneous Equations and the Theory of Production," Econometrica, Vol. 12, pp. 143-205.

Marshal1, A. I. Olkin (1979); Inequalities: Theory of Iajorization and Its Applications, Academic Press. 
Milgrom, P. (1981); "Good News and Bad News: Representation Theorems and Applications," the Bell Journal of Economics, Vol. 12, pp. 380-391.

Miller, R. (1984); "Job Matching and Occupational Choice," the Journal of Political Economy, Vol. 92, pp. 390-409.

Neuendorf, D., R. Shaffer (1987); "Private Sector Econonic Changes in Dane County: 1978-1986," Department of Igricultural Econonics, University of Visconsin.

Pakes, 1. (1986); "Patents as Options: Some Estimates of the Value of Holding European Patent Stocks," Econometrica, Yol. 54, pp. 755-784.

Robinson, P. (1988); "Root-N Consistent Semiparametric Regression," Econometrica, Vol. 56, pp. 931-954.

Ross, S. (1983); Introduction to Stochastic Dynamic Programming, New York and London, Academic Press.

Rust, J. (1987); "Optimal Replacenent of GrC Bus Engines: In Empirical Model of Harold Zurcher," Econometrica, Yol. 55, pp. 999-1034.

Smart, D. (1974); Fixed Point Theorems, Cambridge, Cambridge University Press.

Vedervang, F. (1965); The Development of a Population of Industrial Firms, Scandinavian University Books. 
Volpin, K. (1984); "An Estimable Dynamic Stochastic Model of Fertility and Child Mortality," the Journal of Political Economy, Vol. 92, pp. 852-874.

Zellner, A., J. Kmenta, J.Dreze (1966); "Specification and Estimation of Cobb-Douglas Production Functions," Econonetrica, Vol. 34, pp. 784-95. 


\section{Appendices}

The three appendices provide results used in Sections 2,3 , and 4 respectively. Lemmas, equations, etc. are numbered in a separate consecutive orderring within each appendix.

\section{Appendix I. Proofs of Results Osed in Section 2.}

Part 1. Theorem 2.7 (existence and montonicity of the value function)

At each $t$ there exists a unique $V_{t}(\cdot): N^{t} \rightarrow R_{+}$which provides the value of continuing in operation assuming optimal behavior in each future period. $r_{t}(\cdot)$ is bounded, satisfies $(6)$, and is nondecreasing in $n^{t}$; i.e., if $n_{1}^{t}$ ? $n_{2}^{t}$, then $V_{t}\left(n_{1}^{t}\right) \geq V_{t}\left(n_{2}^{t}\right)$ [for $n^{t} \in N^{t}$, and all $\left.t\right]$.

The proof proceeds as follows. First it considers the finite horizon problem in which a firm which remains active until period $T$ must liquidate for Jollars at $T+1$. For this problem the value of continuing in operation from period $t$ (as a function of past $\eta$-realizations) will be denoted by $\mathrm{r}_{t}^{\mathrm{T}}(\cdot): \mathrm{x}^{\mathrm{t}} \rightarrow \mathbb{R}_{+}$, and the resulting stopping function by $x_{t}^{\mathrm{T}}(\cdot): \mathrm{x}^{\mathrm{t}}$ $-\{0,1\} . \quad V_{t}^{\mathrm{T}}(\cdot)$ can be determined by backuard recursion from the terminal year and a stopping policy which dictates liquidation if and only if the value of continuing in operation is less than . The implied stopping function, $x_{t}^{T}\left(n^{t}\right)$, is one if and only if $n^{t} \in \Delta_{t}^{T}=\left\{n^{t}: v_{1}^{T}\left(n_{1}^{t}\right)>1\right.$, $\left.V_{2}^{T}\left(n_{1}^{t}, n_{2}^{t}\right)>, \ldots, V_{t}^{T}\left(n^{t}\right)>d\right\}$. Is $T$ increases $V_{t}^{T}(\cdot)$ converges (pointwise) to a limit function, $v_{t}(\cdot)$. This limit function is bounded, monotonic in $n^{t}$, and satisfies the Bellman condition, i.e. equation 2.6, in the text. The proof concludes by showing that $v_{t}(\cdot)$, and the associated limit 
stopping policy, $\chi_{t}(\cdot)$, are indeed the solution to the infinite horizon problem.

$\underline{11 \text { Lemma }} P_{\eta_{t+1}}\left(\cdot \mid n_{1}^{t}\right)>P^{P} \eta_{t+1}\left(\cdot \mid n_{2}^{t}\right)$ whenever $n_{1}^{t} \geq n_{2}^{t} \quad\left(n_{1}^{t}, n_{2}^{t} \in N\right.$, and all $\left.t\right)$

Proof Take any $z \in N$. Then $P_{\eta_{t+1}}\left(z \mid n^{t}\right)=\int P_{\eta_{t+1}}\left(z \mid n^{t}, \theta\right) P_{\theta}\left(d \theta \mid n^{t}\right)$.

$P_{\eta_{t+1}}\left(z \mid n^{t}, \theta\right)$ is nonincreasing in $n^{t}$ by (3.i) and strictly decreasing in $\theta$ by (3.ii), while $P_{\theta}\left(\cdot \mid n^{t}\right)$ is stochastically increasing in $n^{t}$ by (4) [ ]

A2 Lemma Fix any $T$ then, $v_{t}^{\mathrm{T}}\left(\mathrm{n}_{1}^{\mathrm{t}}\right) \geq v_{t}^{\mathrm{T}}\left(\mathrm{n}_{2}^{\mathrm{t}}\right)$ whenever $\mathrm{n}_{1}^{\mathrm{t}} \geq \mathrm{n}_{2}^{\mathrm{t}}\left(\mathrm{n}_{1}^{\mathrm{t}}, \mathrm{n}_{2}^{\mathrm{t}}, \epsilon \mathrm{K}^{\mathrm{t}}\right.$, and $t \leq \mathrm{T})$.

Proof The proof is by backward induction on $t$. Note that

$$
V_{T}^{T}\left(n^{T}\right)=\int \pi(\zeta){ }_{\eta_{T}+1}\left(d \zeta \mid n^{T}\right)+\beta \pi
$$

which is nondecreasing in $n^{\mathrm{T}}$ by virtue of the monotonicity of $\pi(\cdot)$, and $\mathrm{A}$. Nor assume monotonicity at $t+1$. Then if $n_{1}^{t} \geq n_{2}^{t}$

$$
\begin{aligned}
\mathrm{V}_{\mathrm{t}}^{\mathrm{T}}\left(\mathrm{n}_{1}^{\mathrm{t}}\right) & =\int \pi(\zeta) \mathrm{P}_{\eta_{\mathrm{t}+1}}\left(\mathrm{~d} \zeta \mid \mathrm{n}_{1}^{\mathrm{t}}\right)+\beta \int \max \left[\mathrm{l}, \mathrm{V}_{\mathrm{t}+1}^{\mathrm{T}}\left(\zeta, \mathrm{n}_{1}^{\mathrm{t}}\right)\right] \mathrm{P}_{\eta_{\mathrm{t}+1}}\left(\mathrm{~d} \zeta \mid \mathrm{n}_{1}^{\mathrm{t}}\right) \\
& \geq \int \pi(\zeta) \mathrm{P}_{\eta_{\mathrm{t}+1}}\left(\mathrm{~d} \zeta \mid \mathrm{n}_{2}^{\mathrm{t}}\right)+\beta \int \max \left[, \mathrm{V}_{\mathrm{t}+1}^{\mathrm{T}}\left(\zeta, \mathrm{n}_{1}^{\mathrm{t}}\right)\right] \mathrm{P}_{\eta_{\mathrm{t}+1}}\left(\mathrm{~d} \zeta \mid \mathrm{n}_{2}^{\mathrm{t}}\right) \\
& \geq \int \pi(\zeta) \mathrm{P}_{\eta_{\mathrm{t}+1}}\left(\mathrm{~d} \zeta \mid \mathrm{n}_{2}^{\mathrm{t}}\right)+\beta \int \max \left[\mathrm{V}, \mathrm{V}_{\mathrm{t}+1}^{\mathrm{T}}\left(\zeta, \mathrm{n}_{2}^{\mathrm{t}}\right)\right] \mathrm{P}_{\eta_{\mathrm{t}+1}}\left(\mathrm{~d} \zeta \mid \mathrm{n}_{2}^{\mathrm{t}}\right)=\mathrm{V}_{\mathrm{t}}^{\mathrm{T}}\left(\mathrm{n}_{2}^{\mathrm{t}}\right),
\end{aligned}
$$


where the inequalities are due to $\mathbf{\Delta 1}$, the monotonicity of $x(\cdot)$, and the hypothesis of the inductive argument.

13 Lemma Fix $T$. Then, $v_{t}^{T+1}\left(n^{t}\right) \geq V_{t}^{T}\left(n^{t}\right) \quad\left(n^{t} \in \mathbb{N}^{t}\right.$, and $\left.t \leq T\right)$

Proof. The proof is again by backvard induction on $t$. For the initial condition of the inductive argument, note that

$$
\begin{aligned}
V_{\mathrm{T}}^{\mathrm{T}+1}\left(\mathrm{n}^{\mathrm{T}}\right) & =\int \pi(\zeta) \mathrm{P}_{\eta_{\mathrm{t}+1}}\left(\mathrm{~d} \zeta \mid \mathrm{n}^{\mathrm{T}}\right)+\beta \int \max \left[\mathrm{s}, \mathrm{V}_{\mathrm{T}+1}^{\mathrm{T}+1}\left(\zeta, \mathrm{n}^{\mathrm{T}}\right)\right] \mathrm{P}_{\eta_{\mathrm{t}+1}}\left(\mathrm{~d} \zeta \mid \mathrm{n}^{\mathrm{T}}\right) \\
& \geq \int \pi(\zeta) \mathrm{P}_{\eta_{\mathrm{t}+1}}\left(\mathrm{~d} \zeta \mid \mathrm{n}^{\mathrm{T}}\right)+\beta=\mathrm{V}_{\mathrm{T}}^{\mathrm{T}}\left(\mathrm{n}^{\mathrm{T}}\right) .
\end{aligned}
$$

Assuming the condition is true for $a=t+1$ we have

$$
\begin{aligned}
V_{t}^{T+1}\left(n^{t}\right) & =\int x(\zeta) P_{\eta_{t+1}}\left(d \zeta \mid n^{t}\right)+\beta \int \max \left[\zeta, V_{t+1}^{T+1}\left(\zeta, n^{t}\right)\right] P_{\eta_{t+1}}\left(d \zeta \mid n^{t}\right) \\
& \geq \int x(\zeta) P_{\eta_{t+1}}\left(d \zeta \mid n^{t}\right)+\beta \int \max \left[S, V_{t+1}^{T}\left(\zeta, n^{t}\right)\right] P_{\eta_{t+1}}\left(d \zeta \mid n^{t}\right)=V_{t}^{T}\left(n^{t}\right) .[]
\end{aligned}
$$

\section{Proof of Theorem 2.7}

Lemma $\Delta 3$ insures that for each $\left(t, n^{t}\right)$ the limit,

$$
V_{t}\left(n^{t}\right)=\lim _{T \rightarrow \infty} V_{t}^{T}\left(n^{t}\right)
$$

exists. Let $\sup _{\eta \in \mathbb{N}} \pi(\eta)=\bar{\pi}[\bar{x}$ exists and is finite by virtue of the compactness of $N$ and the continuity of $x(\cdot)]$. It is straightforward to show that $\mathrm{r}_{\mathrm{t}}^{\mathrm{T}}(\cdot)$ is bounded, uniformly over $t$, by the constant function $(1-3)^{-1} \max [\bar{\pi}, 9$. Since boundedness and (weak) monotonicity are preserved 
by limit functions, this insures that $V_{t}\left(n^{t}\right)$ is monotonic and bounded. Also

$$
\begin{aligned}
\lim _{T \rightarrow \infty} V_{t}^{T}\left(n^{t}\right) & =\int \pi(\zeta) P_{\eta_{t+1}}\left(d \zeta \mid n^{t}\right)+\beta \lim _{T+\infty} \int \max \left[s, V_{t+1}^{T}\left(\zeta, n^{t}\right)\right] P_{\eta_{t+1}}\left(d \zeta \mid n^{t}\right) \\
& =\int \pi(\zeta) P_{\eta_{t+1}}\left(d \zeta \mid n^{t}\right)+\beta \int \max \left[t, V_{t+1}\left(\zeta, n^{t}\right)\right] P_{\eta_{t+1}}\left(d \zeta \mid n^{t}\right),
\end{aligned}
$$

because

$$
\begin{aligned}
& \lim _{\mathrm{T} \rightarrow \infty} \int \max \left[, v_{t+1}^{\mathrm{T}+1}\left(\zeta, n^{\mathrm{t}}\right)\right] P_{\eta_{\mathrm{t}+1}}\left(\mathrm{~d} \zeta \mid \mathrm{n}^{\mathrm{t}}\right) \\
= & \int \lim _{\mathrm{T} \rightarrow \infty} \max \left[\mathrm{l}, \mathrm{V}_{\mathrm{t}+1}^{\mathrm{T}+1}\left(\zeta, \mathrm{n}^{\mathrm{t}}\right)\right] \mathrm{P}_{\eta_{\mathrm{t}+1}}\left(\mathrm{~d} \zeta \mid \mathrm{n}^{\mathrm{t}}\right)
\end{aligned}
$$

by the Lebesgue dominated convergence theorem, since $\left\{\max \left[\phi, V^{T+1}\left(\zeta, n^{t}\right)\right\}\right.$ is dominated by $\max \left(\phi,(1-\beta)^{-1} \bar{x}\right)$ which is integrable with respect to $P_{\eta_{t+1}}\left(\cdot \mid n^{t}\right)$.

be have shown that if $\mathrm{V}_{\mathrm{t}}(\cdot)$, and the associated stopping policy, were optimal, then they would satisfy the conditions of the theorem. What remains is to show that they are indeed optional. To see this assume, to the contrary, that there exists an alternative stopping policy, say $\left\{\chi_{\tau}^{*}(\cdot)\right\}_{\tau=0}^{x}$, where $\chi_{\tau}^{*}\left(n^{\top}\right)$ is one if a firm with $\eta$ realizations of $n^{\top}$ is in operation in period $\tau$ and zero otherwise, which generates a value function, say $V_{t}^{*}\left(n^{t}\right)$, which satisfies, for at least one $\left(t, n^{t}\right)$,

$$
V_{t}^{*}\left(n^{t}\right)-V_{t}\left(n^{t}\right) \geq \epsilon>0 \text {. }
$$


$V_{t}^{*}\left(n^{t}\right) \leq E_{t}\left\{\sum_{\tau=0}^{T} \beta^{\tau}\left[x_{t+\tau}^{*}\left(\eta^{t+\tau}\right) \pi\left(\eta_{t+\tau}\right)+\left\{x_{t+\tau}^{*}\left(\eta^{t+\tau}\right)-x_{t+\tau-1}^{*}\left(\eta^{t+\tau-1}\right)\right\}\right]+\beta^{T} \bar{\tau} /(1-\beta)\right.$ (A5)

$$
\equiv \mathrm{V}_{\mathrm{t}}^{*} \mathrm{~T}\left(\mathrm{n}^{\mathrm{t}}\right)+\beta^{\mathrm{T}} \bar{x} /(1-\beta) \leq \mathrm{V}_{\mathrm{t}}^{\mathrm{T}}\left(\mathrm{n}^{\mathrm{t}}\right)+\beta^{\mathrm{T}} \bar{x}(1-\beta)^{-1} \leq \mathrm{V}_{\mathrm{t}}\left(\mathrm{n}^{\mathrm{t}}\right)+\beta^{\mathrm{T}} \overline{\mathrm{r}}(1-\beta)^{-1},
$$

where $v_{t}^{*} \mathrm{~T}(\cdot)$ is the value function that arises when the policy $\left\{x_{T}^{*}(\cdot)\right\}$ is followed for a $T$-horizon problem. The first inequality follows from the fact that current returns are bounded by $\bar{\pi}$, the second from the fact that $V_{t}^{T}\left(n^{t}\right)$ is the optimum for the $T$ horizon problem, and the third is from $\Delta 3$. Provided $T$ is chosen to be greater than $-\ln [\epsilon(1-\beta) / \pi] /-\ln \beta$, equations 14 and 15 contradict one another.

\section{Part 2. Theorem 2.11 (conditional distributions for $\eta_{t}$ )}

Let $t$ and $k$ be positive intergers with $t>k$, and $\left(i_{1}, \ldots, i_{k}\right)$ be any selection of $k$ distinct elements from $\{1, \ldots, t-1\}$. Then if $\underset{\sim 1}{n_{1}^{\prime}}=$ $\left(n_{i_{1}}^{1}, \ldots, n_{i_{k}}^{1}\right)$ and $n_{n_{2}}^{\prime}=\left(n_{i_{1}}^{2}, \ldots, n_{i_{k}}^{2}\right)$ are arbitrary $\left(i_{1}, \ldots i_{k}\right)$ histories of $\eta$ satisfying $\underset{\sim 1}{n_{1}}>{\underset{\sim}{n} 2}_{2}$, and $\chi_{t}$ is defined as in (2.8),

$$
P_{\eta_{t}}\left(\cdot \mid n_{\sim}, x_{t}=1\right)>_{s} P_{\eta_{t}}\left(\cdot \mid n_{\sim}, x_{t}=1\right)
$$

Proof. For any $z \in \mathbb{N}$ and $\underset{\sim}{n}={\underset{\sim}{n}}_{1}$ or ${\underset{\sim}{n}}_{2}$,

$$
\mathrm{P}_{\eta_{\mathrm{t}}}\left(\left.\mathrm{z}\right|_{\sim}, x_{\mathrm{t}}=1\right)=\int \mathrm{P}_{\eta_{\mathrm{t}}}\left(z||_{\sim}, x_{\mathrm{t}}=1, \theta\right) \mathrm{P}_{\theta}\left(\mathrm{d} \theta \mid \underset{\sim}{\mathbf{n}}, x_{\mathrm{t}}=1\right)
$$

where

$$
P_{\eta_{t}}\left(z \mid n_{n}, \lambda_{t}=1, \theta\right)=\int P_{\eta_{t}}\left(z \mid \eta^{t-1}, \theta\right) P_{\eta_{t-1}}\left(d \eta^{t-1} \mid \underset{N}{n}, x_{t}=1, \theta\right)
$$


Nor use Bayes law to show that for $\eta^{t-1} \geq \eta_{*}^{t-1}$,

$$
\begin{aligned}
& p\left(\left.\eta^{t-1}\right|_{\sim_{1}}, \theta\right) p\left(\left.\eta_{*}^{t-1}\right|_{\sim 2}, \theta\right)-p\left(\left.\eta^{t-1}\right|_{\sim 2}, \theta\right) p\left(\eta_{*}^{t-1} \mid n_{\sim}, \theta\right) \\
& =k\left[p\left(\sim_{1} \mid \eta^{t-1}, \theta\right) p\left({\underset{\sim}{n} 2}_{2} \mid \eta_{*}^{t-1}, \theta\right)-p\left({\underset{\sim}{1} 1}_{1} \mid \eta_{*}^{t-1}, \theta\right) p\left({\underset{\sim}{2}}_{2} \mid \eta^{t-1}, \theta\right)\right] \geq 0,
\end{aligned}
$$

where the inequality is a trivial consequence of $\underset{\sim}{n}$ being deternined by $\eta^{t-1}$. Since conditioning on $\eta^{t} \in \Lambda_{t}=\left\{\eta^{t}: x_{t}\left(\eta^{t}\right)=1\right\}$ does not affect the $\ell$-ordering, we have

$$
P_{\eta_{t}}\left(\cdot \mid \prod_{\sim}, x_{t}=1, \theta\right) \gtrsim_{\ell \Gamma W} P_{\eta_{t}}\left(\cdot \mid \prod_{2}, x_{t}=1, \theta\right) .
$$

Given $(A 6),(17)$ and lemma 2 , the theorem requires only that $\mathrm{P}_{\theta}\left(\cdot \mid \pi_{1}\right) \geq_{t} \mathrm{P}_{\theta}\left(\left.\cdot\right|_{\Omega_{2}}\right)$. But by lemma 4 , this condition is satisfied provided

$$
P_{n}\left(\cdot \mid \theta_{1}\right)>>_{\ell I} P_{n}\left(\cdot \mid \theta_{2}\right)
$$

whenever $\theta_{1}>\theta_{2}$. Take any $\underset{\sim}{n_{1}}>{\underset{\sim}{n}}_{2}$, then

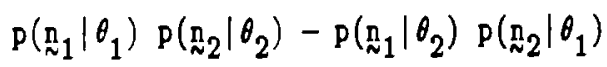

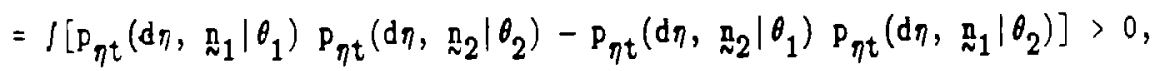

where the integral runs over those $\eta_{t}$ whose indices are in $\{1, \ldots, t-1\}$, but are not in $\left\{i_{1}, \ldots, i_{k}\right\}$, and the inequality results from $(3.11)$. 


\section{Appendix 2. Proofs of Results Osed In Section 3}

Part 1. Theorem 3.2 (properties of the active exploration model)

$\triangle$ unique optimal policy and associated value function exist and they have the following characteristics:

i) $V(\omega)$ is bounded and nondecreasing in $\omega$.

ii) The optimal policy, $x_{\tau}^{*}\left(J_{\tau}\right)$ is bounded, depends only on current $w$, and is stationary, i.e. for all $\tau$

$$
\mathrm{x}_{\tau}^{*}\left(\mathrm{~J}_{\tau}\right)=\mathrm{x}_{\tau}^{*}\left(\omega_{\tau}\right)=\mathrm{x}^{*}\left(\omega_{\tau}\right) \leq \overline{\mathrm{x}}<\boldsymbol{\infty}
$$

iii) There exists a couple, $(\underline{\underline{\omega}}, \overline{\bar{\omega}})$ with, $-\omega<\underline{\underline{\omega}} \leq \overline{\bar{\psi}}<\infty$, such that

$$
x^{*}\left(u^{\prime}\right)=0 \text { if } \omega \notin\left\{w^{\prime}: \underline{\underline{\omega}} \leq \omega^{\prime} \leq \overline{\bar{\psi}}\right\} .
$$

iv) There exists a second couple $(\underline{\underline{\omega}}, \bar{\omega})$, with $\rightarrow<\underline{\underline{w}} \leq \underline{\underline{\omega}} \leq \overline{\bar{\omega}} \leq \bar{\omega}<\boldsymbol{\alpha}$, such that

$$
V(\omega)>\text { if and only if } \omega>\underline{u},
$$

and

$$
\inf _{\mathrm{t}}\left[\underset{u_{0}^{\prime} \leq \bar{u}^{\prime}}{\inf } \operatorname{Pr}\left\{u_{t} \leq \bar{\omega} \mid \omega_{0}\right\}\right]=1 .
$$


Proof: By 1 ssumption 3.1 , the model has a stationary Markovian structure. Hence an optimal policy, if one exists, can be chosen from the class of Yarkov policies [Dynkin and Yushkevich (1975), p. 148]. Existence and properties (i) and (ii) are an immediate consequence of Theorem 5 of Blackwell (1965). That is, let $\nabla \in \ell_{\infty}(I)$ and define an operator $T$ : $\ell_{\infty} \rightarrow \ell_{\infty}$ pointwise as follows:

$$
T U(\omega) \equiv \max \left\{\sup _{x \geq 0}\left(\int_{\eta} \mathbb{R}(\eta, \omega, x) P_{\eta}(d \eta \mid \omega)+\beta \Sigma_{\tau} \sigma(\omega+\tau) P_{\tau}(\tau \mid x)\right), \quad\right\},
$$

where $P_{\tau}$ is the density of $P_{\tau}$. It is straightforward to show that $I$ is a monotone contraction operator, so that the Banach Fixed Point Theorem [D.R. Smart (1974), p. 2-3] gives existence of a unique monotone function $V: \mathbb{l} \rightarrow \mathbb{R}$, uniformly bounded, and satisfying $V=T V$. That $V$ is nondecreasing in $u^{\prime}$ follows from the monotonicity of $\pi(\eta)$ and $c(\omega)$ and the dominance properties of the families $\mathbb{P}_{\eta}$ and $\mathbb{P}_{\tau}(3.1 . i, 3.1 . i i)$. $\Delta S V(\omega)$ is uniformly bounded both above and below, investment $x^{*}(\omega)$ must also be bounded:

$\forall u^{\prime}, x^{*}\left(u^{\prime}\right) \in[0, \bar{x}]$. That $x^{*}(u)$ is unique follows from the strict concavity of the optimand in (A1) in $x$ [Assumption 3.1.iii].

Since the optimal policy solves the pointwise optimization (A1) we have the first order conditions

$$
\begin{gathered}
\left\{c(u)-\beta \underset{\tau}{\Sigma}[V(u+\tau)-V(u)] \frac{\partial}{\partial x} p_{\tau}(\tau \mid x)\right\} \cdot x=0 \\
\{V(u)-1\} \cdot[1-\chi(u)]=0 .
\end{gathered}
$$

To show property (iii) we note that $x^{*}(\omega)>0$ iff $\Sigma\left\{\left(\omega^{\prime}-\tau\right)-V(u) ? \frac{\partial}{\partial x} P_{\tau}(\tau \mid x)\right\}>c(\omega) \beta^{-1}$, and that $c(\omega)$ is bounded away from 
zero (3.1.iii). Let $\Omega_{u}^{\epsilon}=\{\omega \mid \nabla-V(\omega)<\epsilon\}$ and $\Omega_{\ell}^{\epsilon}=\{\omega \mid V(\omega)-i<\epsilon\}$, where $F=$ sup $V\left(\omega^{\prime}\right)$. These are clearly nonempty since $V(u)$ is monotonic, = $\inf V(\omega)=\lim _{\omega \rightarrow \infty} V(\omega)$ and $V=\lim _{\omega \rightarrow \infty} V(\omega)$. If $\omega \in \Omega_{\ell}^{\epsilon}$ then all $\omega^{\prime}<\omega$ are also contained in $\Omega_{\ell}^{\epsilon}$, and similarly $\omega \in \Omega_{u}^{\epsilon}$ implies that all $\omega^{\prime} \geq \omega$ are contained in $\Omega_{u}^{t}$; hence each of these sets contains infinitely many states. Ve will show that $\sum[V(\omega+\tau)-V(\omega)] \frac{\partial}{\partial x} p_{\tau}(\tau \mid x)$ can be made arbitrarily small in both sets, implying that investment must optinally cease.

The boundaries, $\underline{\underline{\omega}}-1 \in \Omega_{\ell}^{\epsilon}$ and $\overline{\bar{\nu}}+1 \in \Omega_{u}^{\epsilon}$, for some generally different $\epsilon$ 's, will be the highest, respectively - lowest, $\omega$ in those sets such that $\sum_{\tau}[V(\omega+\tau)-Y(\omega)] \frac{\partial}{\partial x} \mathrm{p}_{\tau}(\tau \mid x)<\frac{c(\omega)}{\beta}$. To complete this argument we show that the l.h.s. of this expression can be made arbitrarily small on $\Omega_{u}^{\epsilon}$. An identical argument, mutatis mutandus, can be used to show the same for $\Omega_{\ell}^{\epsilon}$. Note that compact support for $\mathbb{P}_{\tau}, T,[\Lambda$ ssumption 3.1.ii] implies that there exists an $\bar{\psi} \in \Omega_{u}^{\epsilon}$ such that $\forall w \geq \bar{u}, P_{\tau}\left\{\mathbb{Z} \backslash \Omega_{u}^{\epsilon} \mid x\right\}=0 \forall x \geq 0$, i.e. the transition probability puts zero weight on states not in $\Omega_{u}^{\epsilon}$. Then

$$
\left.\sum_{\tau \in \mathrm{T}}[V(\omega+\tau)-V(\omega)] \frac{\partial}{\partial \mathrm{x}} \mathrm{p}_{\tau}(\tau \mid \mathrm{x})\right\} \leq \epsilon .
$$

Hence the impact of investment on future expected returns becomes arbitrarily small as $\omega$ increases in $\Omega_{u}^{\epsilon}$, so we can find a boundary above which no investment will optimally take place. Let $\overline{\bar{u}}$ the lowest of such boundaries. Similarly we can find a greatest $w \in \Omega_{l}^{\epsilon}$ such that $x(\omega)=0$, which we label $\underline{\underline{\omega}}$.

To show property (iv) we need only note that $x(\omega)=0$ implies $P_{\tau}\{0 \mid 0\}=1$ so that once $u_{t} \leq \underline{\underline{\omega}}$ for any $\mathrm{t}, \forall s \geq \mathrm{t} \omega_{\mathrm{s}} \leq \underline{\underline{\omega}}$. Since $3.1 . \mathrm{i}$ insures there exists an $\omega^{*}$ such that for all $\omega<\omega^{*}, \int \pi(\eta) P_{\eta}(d \eta \mid \omega) \leq$ $(1-3)^{-1}$, for $\omega \leq \min \left(\omega^{*}, \underline{\underline{\omega}}\right)$ 


$$
V(\omega)=\max \left\{\int_{\eta} \pi(\eta) P_{\eta}\left(d_{\eta} \mid \omega\right)+\beta \sum_{\tau \leq 0} V(\omega+\tau) P_{\tau}(\tau \mid 0), \boldsymbol{l}\right\}=\mathbf{I}
$$

Let $\underline{w}$ be the greatest such $\omega \leq \underline{\underline{w}}$ at which $\mathbf{1 3}$ holds. To show existence of $\bar{w}$, let $\mathrm{k}=\#\{$ supp $\mathrm{T}\} . \quad \Delta \mathrm{P}_{\tau}\{0 \mid 0\}=1, \bar{w} \leq \overline{\bar{w}}+\mathbf{k}$ nust surely hold since the probability of transfering to a higher $u$ from any $\omega^{\prime}>\overline{\bar{u}}$ is zero, and the highest w potentially achievable from $\omega^{\prime} \leq \overline{\bar{\omega}}$ is $\omega^{\prime}+\mathbf{k}$. Hence, $\forall \omega_{0} \leq \bar{\omega}$, $\operatorname{Pr}\left\{\omega_{1} \leq \bar{w} \mid \omega_{0}\right\}=1$, and by induction $\operatorname{Pr}\left\{\omega_{t} \leq \bar{v} \mid \omega_{0}\right\}=1$, where $\omega_{t}$ is the state following t transitions. Therefore

$$
\inf _{t}\left\{\inf _{u_{0} \leq \bar{w}} \operatorname{Pr}\left\{u_{t} \leq \bar{w} \mid u_{0}^{\prime}\right\}\right\}=1
$$

\section{Part 2. Lemma 3.6 (-mixing of the $\left\{\mathrm{S}_{\mathrm{t}}^{\mathrm{a}}\right\}$ process).}

Let $\left\{S_{t}^{a}\right\}_{t=1}^{\infty}$ be the stochastic process formed from the distribution of sales conditional on survival and any initial $\omega_{0} \in\{1,2, \ldots, \mathbf{K}\}$, and $\mathbf{y}_{x}^{y}$ be the $\sigma$-algebra generated by possible realizations of $\mathrm{S}_{\mathrm{x}}^{\mathrm{a}}, \mathrm{S}_{\mathrm{x}+1}^{\mathrm{a}}, \ldots, \mathrm{S}_{\mathrm{y}}^{\mathrm{a}}$. Then $\left\{\mathrm{S}_{\mathrm{t}}^{\mathrm{a}}\right\}$ $\phi$-mixes at a geometric rate, i.e.

$$
\sup \left\{\left|P\left(E_{2} \mid E_{1}\right)-P\left(E_{2}\right)\right|, E_{1} \text { with } P\left(E_{1}\right)>0 \text { and } E_{1} \in \mathbf{Y}_{1}^{X}, E_{2} \epsilon \mathbf{Y}_{x+\tau}^{\infty}\right\} \leq \Delta \phi^{\tau}
$$
with $\phi<1$.

Proof Briefly, the probability space for this process is constructed from the finite set $\underline{S}=\{S: S=S(\eta), \eta \in \mathbb{N}\}$, the family of probability measures for $\eta, \mathbb{P}_{\eta}$, and the Yarkov transition matrix for $\omega, \underline{p}$ as follows. The sample space consists of the set of all possible infinite sequences of elements

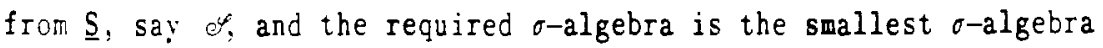
containing $e^{\circ}$ Let $Q$ be formed from $\underline{p}$ by dividing its $i^{\text {th }}$ row by $1-p_{i}, 0$ 
(for $i=1, \ldots, k$ ) and then deleting its first row and column. $Q$ is the Markov transition matrix for $\omega_{t+1}$ conditional on $w_{t}$ and survival until $t+1$. Note that $3.1 . i$ i together with 3.2 insure that this transition matrix is irreducible aperiodic (see Billingsley, 1979, chapter 1). The measures for the alternative sample paths can be computed directly from $Q, P_{\eta}$, and $\omega_{0}$.

To prove the lemma let $S_{x}^{y}=\left\{S_{x}^{a}, \ldots, S_{y}^{a}\right\}$. Then it suffices to show that for any $E_{1} \in \mathbf{I}_{1}^{X}$ such that $P\left(E_{1}\right)>0$, and any $E_{2} \in \mathbf{I}_{X+\tau}^{X+\tau+r}$

$$
\left|P\left\{\left(S_{x+\tau}^{x+\tau+r} \epsilon E_{2}\right) \cap\left(S_{1}^{x} \epsilon E_{1}\right)\right\}-P\left\{S_{x+\tau}^{x+\tau+r} \epsilon E_{2}\right\} P\left\{S_{1}^{x} \in E_{1}\right\}\right| \leq \Delta \phi^{\tau}
$$

(Billingsley 1968, section 20). Since both $E_{2}$ and $E_{1}$ are finite sets we can, without loss of generality, assume that both are singletons. Taking $I=x=1$ for simplicity, and recalling that $S_{k}^{a}=\bar{s}\left(w_{k}\right)+\psi_{k}$ where the distribution of $\mu_{k}$ conditional on $\mathbf{r}_{1}^{k-1} u_{k}$ depends only on $\omega_{k}$, the first probability within the absolute value sign can be written as

$$
\begin{aligned}
& P\left\{\left(s_{\tau+1}^{a}=s_{\tau+1}\right) \cap\left(s_{1}^{a}=s_{1}\right)\right\} \\
& =\sum_{w_{1}} \sum_{\tau+1} P\left\{u_{\tau+1}=s_{\tau+1}-\bar{s}\left(\omega_{\tau+1}=j\right) \mid u_{1}=s_{1}-\bar{s}\left(\omega_{1}=i\right), \omega_{\tau+1}=j, \omega_{1}=i\right\} \\
& P\left\{\omega_{\tau+1}=j \mid u_{1}=s_{1}-\bar{s}\left(\omega_{1}=i\right), \omega_{1}=i\right\} P\left\{u_{1}=s_{1}-\bar{s}\left(\omega_{1}=i\right) \mid \omega_{1}=i\right\} P\left\{\omega_{1}=i\right\} \\
& =\sum_{i=1}^{k} \sum_{j=1}^{k} P_{u}\left(s_{\tau+1}-\bar{s}(j) \mid \omega=j\right) q_{i j}^{(\tau)_{P_{u}}}\left(s_{1}-\bar{s}\left(\omega_{1}=i\right) \mid \omega=i\right) P_{i}^{0},
\end{aligned}
$$

where $\left.q^{(\tau)}\right)_{i, j}$, provides the $\tau$-period transition probabilities from the $Q$ chain. [Recall that $p^{0}$ provides the initial wdistribution]. Since $Q$ is 
irreducible aperiodic it has a unique invariant distribution, say

$q^{* \prime}=\left[q_{1}^{*}, \ldots, q_{k}^{*}\right]$, and

(16)

$$
\max _{(i, j) \in \Omega^{2}}\left|\frac{q_{i j}^{\tau}}{q_{j}^{*}}-1\right| \leq \Delta_{0} \phi^{\tau}
$$

(see Billingsley, 1979, sec. 1.8). Jsing this invariant distribution to evaluate the unconditional probability that $\mathrm{S}_{\tau+1}^{\mathrm{a}}=\mathrm{s}_{\tau+1}$, so that $\mathrm{P}\left(\mathrm{S}_{\tau+1}^{\mathrm{a}}=\right.$ $\left.s_{\tau+1}\right)=\sum_{j=1}^{k} P_{u}\left(s_{\tau+1}-\bar{s}(j) \mid \omega=j\right) q_{j}^{*}$, noting that $P\left(s_{1}^{a}=s_{1}\right)=\sum_{i=1}^{k}$ $P\left(s_{1}-\bar{s}(i) \mid \omega=i\right) p_{i}^{0}$, and substituting $q_{(i, j)}^{\tau}=q_{i, j}^{\tau}-q_{j}^{*}+q_{j}^{*}$ into (15) we have

$$
\begin{aligned}
& \left|P\left\{S_{\tau+1}^{a}=s_{\tau+1} \cap S_{1}^{a}=s_{1}\right\}-P\left\{S_{\tau+1}^{a}=s_{\tau+1}\right\} P\left\{S_{1}=s_{1}\right\}\right| \\
& =\sum_{i=1}^{k} \sum_{j=1}^{k}\left|p_{u}\left(s_{\tau+1}-\bar{s}(j) \mid \omega=j\right) p_{u}\left(s_{1}-\bar{s}(i) \mid \omega=i\right) p_{i}^{0}\left(q_{i j}^{\tau}-q_{j}^{*}\right)\right| \leq \\
& \sum_{i=1}^{k} \sum_{j=1}^{k}\left|q_{i, j}^{\tau}-q_{j}^{*}\right| \leq \Lambda_{0} \phi^{\tau} \sum_{i=1}^{k} \sum_{j=1}^{k} q_{j}^{*} \equiv \Lambda \phi^{\tau},
\end{aligned}
$$

where the last inequality follows from (16).

Appendix 3. The Test Statistics ${ }^{1}$

Ve begin by developing a test for the null hypothesis that

1 The reader interested in more detail on the testing procedures used in this section should consult Barlow et. al. (1972), or the more recent econometric literature on testing subject to inequality constraints which begins with the work of Gourieroux, Holly, and Yonfort (1982). Golberger's (1987) exposition is particularly clear. 


$$
\mathbf{H}_{\mathbf{Y}}^{0}: \mathbf{R}_{\mu}^{\mathbf{X}}=\mathbf{I} \geq 0,
$$

where $B$ has full row rank, say $C$, under the maintained hypothesis that $I \in \mathbb{R}^{C}$. To this end ve consider the following two estinators for $r$

(12a) $\hat{\mathrm{r}}=\hat{\mathrm{R} \mu}$

and

$$
\hat{\mathbf{r}}_{\mathbf{Y}}=\arg \underset{\mathbf{m} \geq 0}{\min }\left[(\mathbf{r}-\hat{\mathrm{r}})^{\prime} R\left[\hat{\mathrm{V}}^{\mathbf{k}}\right]^{-1} \mathbf{R}^{\prime}(\mathbf{r}-\hat{\mathrm{r}})\right]
$$

$\dot{r}$ is an 'unconstrained' estimator of $r$ obtained from substituting sample for population means. $\mathbf{I}$ is a 'constrained' estimator, an estimator forced to satisfy the inequality constraint of the null. Subject to that

constraint, it is obtained by minimizing a quadratic form in $(r-r)$, where the weighting matrix, $\mathbb{R}\left[\hat{\gamma}^{k}\right]^{-1} R$, is chosen to be the estimated variance-covariance of $\hat{I}$ under the hypothesis that $\mathbb{R}_{\mu}^{\mathbf{k}}=0$.

Since the quadratic form in $(\mathbf{A b})$ is nonnegative and equal to zero if $r=\dot{r}$, if $\dot{r} \geq 0, \dot{r}=r$. Figure (4) illustrates possible solutions for $\dot{r}$ in the case where $C=2$. The ellipsoids represent sets of $r$ which produce a constant $(\mathbf{r}-\hat{r})^{\prime} R_{[}\left[\hat{r}^{k}\right]^{-1} R^{\prime}(r-\hat{r})$ value.

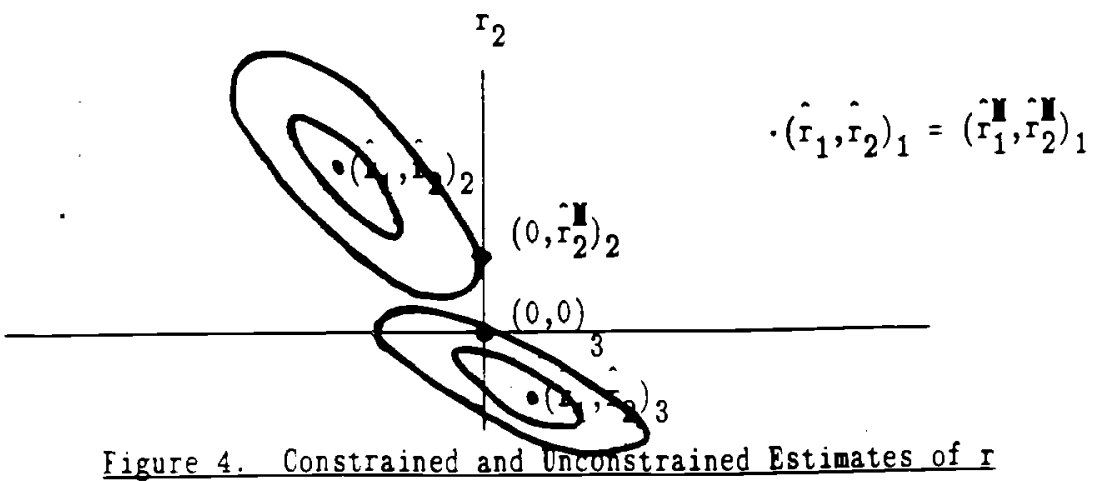




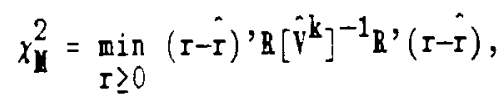

then large realized values of this statistic are evidence against $\mathbf{H}_{\mathbf{M}}^{0}$. Indeed, Barlow, Bartholemew, Bremner, and Brunk (1972) have shown that for al1 a $\geq 0$

(A4a) $\quad T_{\mathbf{Y}}(\mathrm{a})=\operatorname{Pr}\left\{x_{\mathbf{Y}}^{2}>\mathrm{a} \mid \mathrm{r}=0\right\} \rightarrow \sum_{\mathrm{c}=0}^{\mathrm{C}} \mathbf{V}(\mathrm{c}) \operatorname{Pr}\left\{x_{\mathrm{C}}^{2}>\mathrm{a}\right\}$

as sample size grows large, where

$$
W(c)=\operatorname{Pr}\left\{I^{\mathbf{Y}} \text { has exactly c zero components } \mid \mathrm{I}=0\right\}
$$

and $x_{c}^{2}$ denotes a chi-square deviate with precisely $c$ degrees of freedom $(c=0, \ldots, C)$. Thus, if $x_{\mathbf{Y}}^{0}$ is the realized value of $x_{\mathbf{Y}}^{2}, \mathrm{~T}_{\mathbf{Y}}\left[x_{\mathbf{Y}}^{0}\right]$ provides the "p-value" (or the probability of type I error) of a test that would reject the null if $\chi_{\mathbf{Y}}^{2}=\chi_{\mathbf{Y}}^{0}$ when the true value of $\mathbf{r}$ was zero. The $p-v a l u e$ when $r$ is any value greater than zero cannot be larger.

Unfortunately, the orthant probabilities, that is the values of $\{\mathrm{H}(\mathrm{c})\}_{\mathrm{C}=0}^{\mathrm{C}}$ needed to obtain $(\mathbf{1 4 a})$, are difficult to calculate. As a result we obtain simulated estimates of their values, say $\hat{V}_{c}$, and provide a simulated estimate of $\mathrm{T}_{\mathbf{I}}(\cdot)$ say $\dot{\mathrm{T}}_{\mathbf{Y}}$, where

$$
\hat{T}_{\mathbf{Y}}[\mathrm{a}]=\sum_{\mathrm{C}=0}^{\mathrm{C}} \hat{\mathrm{H}}_{\mathrm{c}} \operatorname{Pr}\left\{\chi_{c}^{2}>\mathrm{a}\right\}=\hat{\mathbf{Y}}^{\prime} \mathbf{X}
$$

and

$$
\hat{\mathbf{H}}=\left[\hat{\mathrm{H}}_{0}, \ldots, \hat{\mathbf{V}}_{\mathrm{C}-}\right] \text {, whereas } \boldsymbol{X}^{\prime}=\left[\mathrm{P}_{\mathrm{I}}\left\{x_{0}^{1}>\mathrm{a}\right\}, \ldots, \operatorname{Pr}\left\{x_{\mathrm{C}}^{2}>\mathrm{a}\right\}\right] .
$$


Since the $\hat{V}_{c}$ can be regarded as cell means from repeated draws from a multinomial distribution (where NSIK, the number of simulations, is the number of draws), the variance of $\hat{T}_{\mathbf{Y}}$ [a] about its expected value of $T_{\mathbf{I}}[\mathrm{a}$ ] can be obtained from the formula for the rariance of a nultinomial as;

$$
\operatorname{Var}\left[\dot{T}_{\mathbf{Y}}(\mathrm{a})\right]=\mathbf{X}^{\prime}\left[\operatorname{diag} \mathbf{V}-\mathbf{W}^{\prime}\right] \mathbf{I}(\mathbf{M S I})^{-1}
$$

So, along with $\hat{\mathrm{T}}_{\mathbf{Y}}(\mathrm{a})$, ve provide an estiate of its variance obtained from substituting the simulated for the actual values of $\mathbf{V}$ in this variance formula.

Next we need a test of

(A7) $\quad \mathbf{H}_{2}^{0}: \quad \mathrm{B}_{\mu}{ }^{k}=\mathrm{r}=0$

under the maintained hypothesis given by $\mathbf{H}_{\mathbf{l}}^{0}$ in $\left(\mathbf{\Delta 1 )}\right.$. Once again $\dot{r}^{\mathbf{M}}$ in (A2b) will serve as our estimate of $\mathbf{r}$ given $\mathbf{H}_{\mathbf{Y}}^{0}$, while under $\mathbf{H}_{Z}^{0}$ the estimate of $I$ is zero (thus, in Figure 4, the ellipsoids bring us from $I$ to the estimator which abides by $\mathbf{H}_{\mathbf{r}}^{0}$, while the dashed lines bring us from the latter to the estimator which abides by $\mathbf{I}_{2}^{0}$ ). I measure of the distance between the estimator obtained conditional on the null and the estimator which is only constrained to satisfy the aintained hypothesis is given by

$$
x_{Z}^{2}=\mathbf{r}^{\mathbf{Y}}{ }_{\mathrm{R}}\left[\hat{\mathrm{V}}^{\mathrm{k}}\right]^{-1} \mathbf{R}^{\prime} \mathbf{r},
$$

Once again, for all a >0 
(19a) $\quad \mathrm{T}_{\mathrm{Z}}(\mathrm{a})=\operatorname{Pr}\left\{x_{Z}^{2}>\mathrm{a} \mid \mathrm{r}=0\right\} \rightarrow \sum_{\mathrm{c}=0}^{\mathrm{C}} \overline{\mathrm{V}}(\mathrm{c}) \operatorname{Pr}\left\{x_{\mathrm{c}}^{2}>\mathrm{a}\right\}$

as sample size grows large, where, in this case

$$
\tilde{V}(c)=\operatorname{Pr}\{\hat{r} \mathbf{Y} \text { has exactly c positive components } \mid \mathbf{r}=0\}
$$

and $x_{c}^{2}$ is defined as above $(c=0,1, \ldots, c)$. Letting $x_{Z}^{0}$ be the observed value of $x_{\eta}^{2}$, we will provide estimates of $T_{Z}\left[x_{Z}^{0}\right]$, say $\dot{T}_{Z}\left[x_{\eta}^{0}\right]$ (obtained from simulating the $\bar{V}(c))$, together with an estimate of the variance of $\hat{T}_{Z}\left[x_{Z}^{0}\right]$.

To compare this sequence of tests, that is the test for weak monotonicity under an unconditional maintained hypothesis coupled with the test of the hypothesis that $s_{1}$ has no effect on the regression function conditional on the maintained that any effect is nondecreasing, to the more familiar direct test of whether $s_{1}$ has no effect on the regression function conditional on an unconstrained maintained hypothesis, note that one test of the latter would check whether

$$
\left.x_{T}^{2}=\hat{I}^{\prime} R_{L} \hat{Y}^{k_{j}}\right]^{-1} R^{\prime} \hat{r}
$$

were close to zero. Under the unconstrained maintained hypothesis $\chi_{\mathrm{T}}^{2}$ has the familiar chi-square distribution with $C$ degrees of freedom. Since the properties of Lagrange multipliers insure that

$$
\left[\mathbf{I}-\mathbf{r}^{\mathbf{Y}}\right]^{\prime} \mathrm{R}\left[\mathrm{V}^{\mathrm{k}}\right]^{-1} \mathbf{R}^{\prime} \mathbf{T}^{\mathbf{Y}}=0
$$

we have from $(12 b)$ and $(18)$, that 


$$
x_{\mathrm{I}}^{2}=\chi_{\mathrm{I}}^{2}+\chi_{Z}^{2}
$$

with probability one. 